\title{
Some lattices of closure systems on a finite set
}

\author{
Nathalie Caspard ${ }^{1}$ and Bernard Monjardet ${ }^{2}$ \\ ${ }^{1}$ LACL, Université Paris 12 Val-de-Marne, 61 avenue du Général de Gaulle, 94010 Créteil cedex, France. \\ E-mail: caspardeuniv-paris12.fr \\ 2 CERMSEM, Maison des Sciences Économiques, Université Paris 1, 106-112, bd de l'Hôpital, 75647 Paris Cedex \\ 13, France. \\ E-mail: monjarde@univ-paris1.fr
}

received May 2003, accepted Feb 2004.

In this paper we study two lattices of significant particular closure systems on a finite set, namely the union stable closure systems and the convex geometries. Using the notion of (admissible) quasi-closed set and of (deletable) closed set we determine the covering relation $\prec$ of these lattices and the changes induced, for instance, on the irreducible elements when one goes from $\mathcal{C}$ to $\mathcal{C}^{\prime}$ where $\mathcal{C}$ and $\mathcal{C}^{\prime}$ are two such closure systems satisfying $\mathcal{C} \prec C^{\prime}$. We also do a systematic study of these lattices of closure systems, characterizing for instance their join-irreducible and their meet-irreducible elements.

Keywords: Anti-exchange closure operator, closure system, convex geometry, (locally distributive) lattice, quasiclosed set.

\section{Introduction}

The notion of closure system (also called Moore family) or the equivalent notions of closure operator or of complete implicational system are fundamental since they very often appear in pure and applied mathematics. For instance, an earlier result in the theory of relational databases has proved the existence of a one-to-one correspondence between the full systems of (functional) dependencies and the closure systems (Armstrong [3]). Then the study of the set of all full systems of dependencies is equivalent to the study of the set all closure systems. In particular, the changes in a full system of dependencies can be studied in terms of changes in a closure system (see for instance [8] and [14]). It has been known for a long time that the set $\mathcal{M}$ of all closure systems defined on a finite set $S$ and ordered by inclusion is a lattice This lattice has been studied by many authors (see, for instance, Caspard and Monjardet [9]).

\footnotetext{
$\dagger$ In 1943, Öre studied the lattice of closure operators in which $\emptyset$ is a fixed point, that is a lattice dual of the lattice of closure systems where $\emptyset$ is closed.

1365-8050 @ 2004 Discrete Mathematics and Theoretical Computer Science (DMTCS), Nancy, France
} 
In this paper we investigate the properties of sub-semilattices of $\mathcal{M}$, formed by the sets of all closure systems satisfying some given significant properties. Namely these sets are the union stable closure systems and the convex geometries. The first one of these sets, ordered by set inclusion, is a lattice, whereas the second one is a join semilattice that we transform into a lattice by adding a least element. We do a systematic study of these lattices. We determine their irreducible elements, their arrow relations and some dependence relations between these irreducible elements. An essential task consists in characterizing the covering relation of these lattices; this problem, easy in $\mathcal{M}$, can be difficult in other cases. It is related — but not equivalent — to the following one: if $\mathcal{L}$ is a class of lattices and $L$ a lattice of $\mathcal{L}$, what are the minimal sets of elements of $L$ which can be deleted from $L$ (respectively, added to $L$ ) in order to obtain a lattice which still belongs to $\mathcal{L}$ ? These questions were investigated in Bordalo and Monjardet [6] in the particular case where a unique element can be deleted or added. Answers were given for several classes of lattices, in particular for the lower locally distributive lattices, those ones whose set representations are the convex geometries. The same question was considered by Chen, Koh and Tan [10] and by Rival [35] [36] and others in their study on Frattini sublattices and on maximal sublattices of distributive lattices, those ones whose set representation is a $T_{0}$-topology. On the other hand, Johnson and Dean [23] prove two theorems on the expansion and the contraction of a quotient of a convex geometry. As we will see their results are contained in our characterizations of the covering relation in the lattice of all convex geometries.

The characterization of the covering relation of our two lattices allows us to determine the changes occuring in such a system when one goes from this system to one covering it or covered by it. In our conclusion, we will come back on the interest of considering these changes.

The basic tools used in this paper to get our results are the notions of quasi-closed set and of $\mathcal{C}$ admissible quasi-closed set, where $\mathcal{C}$ is a given class of closure systems.

\section{Preliminaries}

In the whole paper, all objects are assumed to be finite, so "set" (respectively, "poset", "lattice", etc.) means finite set (respectively, finite poset, finite lattice, etc.). The set difference between two sets $A$ and $B$ is denoted by $A-B$ and by $A-x$ (rather than $A-\{x\}$ ) if $B=\{x\}$. Moreover the symbol + will denote the disjoint set union. We will as well write $A+x$ rather than $A+\{x\}$. At last, we write $A \| B$ when $A$ and $B$ are not comparable (with respect to set inclusion).

A poset $(X, \leq)$ will often be simply denoted by $X$. The associated covering relation will be denoted by $\prec(x \prec y$ if $x \leq z<y$ implies $x=z)$. A (closed) interval of $X$ is a set $[x, y]=\{z \in X: x \leq z \leq y\}$ for some $x$ and $y$ of $X$ satisfying $x \leq y$. An element of a poset $X$ is join-irreducible (respectively, meetirreducible) if it is not the join (respectively, the meet) of elements different from itself. Otherwise it is called join-reducible (respectively, meet-reducible). If the poset is a lattice $L$, meaning that every pair $\{x, y\}$ of elements of $L$ has a join $x \vee y$ and a meet $x \wedge y$, the set of its join-irreducible elements (respectively, of its meet-irreducible elements) will be denoted by $J_{L}$ (respectively, $M_{L}$ ). In this case and as usual $j^{-}$ (respectively, $\mathrm{m}^{+}$) will denote the unique element covered by the join-irreducible $j$ (respectively, the unique element covering the meet-irreducible $m$ ).

We recall the definition of the arrow relations of a lattice $L$ defined on $\left(J_{L} \times M_{L}\right)$ and of the dependence relations $\delta$ and $\beta$. Let $j$ be a join-irreducible and $m$ a meet-irreducible of a lattice $L$. We write $j \uparrow m$ (respectively, $j \downarrow m$ ) if $j \not \leq m$ and $j \leq m^{+}$, i.e. if $j \vee m=m^{+}$(respectively, if $j \not \leq m$ and $j^{-} \leq m$, i.e. if 
$j \wedge m=j^{-}$). We write $j \uparrow m$ if $j \uparrow m$ and $j \downarrow m$ are simultaneously satisfied. Now for $j$ and $j^{\prime} \in J_{L}$, we set $j \delta j^{\prime}$ if there exists $m \in M_{L}$ such that $j \uparrow m$ and $j^{\prime} \not \leq m$. Dually for $m$ and $m^{\prime} \in M_{L}$ we set $m \beta m^{\prime}$ if there exists $j \in J_{L}$ such that $j \downarrow m$ and $j \not \leq m^{\prime}$.

Remark: For the basic properties on the arrow relations, see for instance Ganter and Wille [21]. The dependence relation $\delta$ defined on $J_{L}$ has been introduced by Monjardet [30] in order to study consensus problems in lattices (see [31] for some results on this relation). The relation $\beta$ defined on $M_{L}$ is a dependence relation, dual from the relation $\delta$. There is another dependence relation, denoted by $\delta_{d}$ and defined on $J_{L}$ by $j \delta_{d} j^{\prime}$ if there exists $m \in M_{L}$ such that $j \uparrow m$ and $j^{\prime} \downarrow m$. This relation was introduced by Day [13] in his study of lower bounded lattices. A lattice $L$ is atomistic if and only if $\delta=\delta_{d}$ on $J_{L}$ (see [31]).

A set system on a set $S$ is a family $C$ of subsets of $S$. A closure system $C$ on a set $S$ is a set system on $S$ satisfying the two following properties:

1. $S \in \mathcal{C}$

2. $C_{1}, C_{2} \in \mathcal{C} \Longrightarrow C_{1} \cap C_{2} \in \mathcal{C}$

Example: For any $A \subset S$, we write $\mathcal{C}_{A}$ the set system $\mathcal{C}_{A}=\{A, S\}$ and for all $A, B \subseteq S, \mathcal{C}_{A, B}$ is the set system defined by $\mathcal{C}_{A, B}=\{X \subseteq S: A \nsubseteq X$ or $B \subseteq X\}$. Clearly $\mathcal{C}_{A}$ and $\mathcal{C}_{A, B}$ are closure systems and we will use them later in the paper.

The sets of a closure system $\mathcal{C}$ are called the closed sets of $\mathcal{C}$. A closure system $\mathcal{C}$ is a lattice $(\mathcal{C}, \subseteq, \wedge, \vee)$ (often simply denoted by $\mathcal{C}$ ) with

$$
C_{1} \wedge C_{2}=C_{1} \cap C_{2} \quad C_{1} \vee C_{2}=\bigcap\left\{C \in C: C_{1} \cup C_{2} \subseteq C\right\}
$$

A closure operator $\sigma$ on a set $S$ is a map defined on $P(S)$, which is isotone $(X \subseteq Y \Longrightarrow \sigma(X) \subseteq \sigma(Y))$, extensive $(X \subseteq \sigma(X))$ and idempotent $\left(\sigma^{2}(X)=\sigma(X)\right)$. Equivalently $\sigma$ is a closure operator if we have ( $X \subseteq \sigma(Y)$ if and only if $\sigma(X) \subseteq \sigma(Y)$ ), for all $X, Y \subseteq S$. The fixed points of a closure operator $\sigma$, i.e. the sets $X$ such that $X=\sigma(X)$, are called the closed sets of $\sigma$.

It is well known that closure operators are in a 1-1 correspondence with closure systems by the map associating to a closure operator $\sigma$ the set system $\mathcal{C}_{\sigma}$ of its closed sets and, conversely, by the inverse map associating to a closure system $\mathcal{C}$ the operator $\sigma_{C}$ defined by $\sigma_{C}(X)=\bigcap\{C \subseteq C: X \subseteq C\}$.

We need to recall several classical notions relative to closure systems or closure operators.

Definition 1 Let $\sigma$ be a closure operator on $S$.

1. For $A \subseteq S$, an element $x \in A$ is an extreme element of $A$ (w.r.t. $\sigma)$ if $\sigma(A-x) \subset \sigma(A)$. We denote by ex $\sigma$, or if no ambiguity occurs, by exA the set of all extreme elements of $A$ (w.r.t. to $\sigma)$.

2. A set $A \subseteq S$ is free if $A=e x A$, i.e. if for every $x \in A, x \notin \sigma(A-x)$. A set is dependent if it is not free.

‡ The problem of consensus in a lattice $L$ consists in defining consensus functions on $L$, i.e. functions $F: L^{n} \rightarrow L$ satisfying "good" properties, for instance unanimity $(F(x, \ldots, x)=x)$. The fact that the dependence relations $\delta$ or $\beta$ are - or are not - strongly connected plays a significant role in the latticial theory of consensus, see [27] and [33]. 
3. $A$ basis (or minimal generating set) of a closed set $C$ of $\sigma$ is a subset $B$ of $C$ such that $\sigma(B)=C$ and satisfying $\sigma(B-x) \subset \sigma(B)$ for every $x \in B$.

The following lemma recalls some elementary properties concerning these notions.

Lemma 1 Let $\sigma$ be a closure operator on S. The following holds:

1. For every $A \subseteq S, \emptyset \subseteq \operatorname{ex} \sigma(A) \subseteq \operatorname{exA} \subseteq A \subseteq \sigma(A)$.

2. An element $x$ of a closed set $C$ is an extreme element of $C$ if and only if $C-x$ is closed.

3. $B \subseteq S$ is a basis for a closed set of $\sigma$ if and only if $B$ is free.

4. If $C$ is a closed set of $\sigma$ then exC $=\bigcap\{B: B$ basis of $C\}$.

The following (and less classical) notion of quasi-closed set is fundamental in this paper.

Definition 2 Let $C$ be a closure system on $S$ and $\sigma_{C}$ the associated closure operator. $Q \subset S$ is a quasiclosed set (w.r.t. to $C$ ) if $Q \notin C$ and if $\mathcal{C}+\{Q\}$ is a closure system on $S$. Moreover if $\sigma_{\mathcal{C}}(Q)=C$, we say that $Q$ is a $C$-quasi-closed set.

It will follow from Lemma 4 (given below) that any closure system distinct from $2^{S}$ admits at least one quasi-closed set.

Proposition 1 Let $C$ be a closure system on $S, \sigma_{C}$ the associated closure operator, $Q$ a subset of $S$ with $Q \subset \sigma_{\mathcal{C}}(Q)=C$. The following properties are equivalent:

1. $Q$ is a C-quasi-closed set (w.r.t. $C$ ),

2. For every closed set $G$ satisfying $Q \not \subset G, G \cap Q \in \mathcal{C}+\{Q\}$,

3. For every closed set $G$ such that $G \prec C, G \cap Q \in \mathcal{C}$,

4. For every closed set $G$ such that $G=(C-A) \prec C,(Q-A) \in \mathcal{C}$,

5. For every $X \subset Q$, if $\sigma_{\mathcal{C}}(X) \subset C$ then $\sigma_{\mathcal{C}}(X) \subset Q$.

\section{Proof:}

1. $\Longrightarrow$. Immediate from the definition of a $C$-quasi-closed set.

2, $\Longrightarrow$ 3, $\sigma_{\mathcal{C}}(Q)=C$ and $G \prec C$ imply $Q \not \subset G$ and so $G \cap Q \neq Q$. Thus by item 2, $G \cap Q \in \mathcal{C}$.

3. $\Longrightarrow$ 4. Immediate since $G=C-A$ and $Q \subset C$ imply $G \cap Q=Q-A$.

4] $\Longrightarrow$ 5. Let $X \subset Q$ such that $\sigma_{\mathcal{C}}(X) \subset C=\sigma_{\mathcal{C}}(Q)$. Then there exists $G \in \mathcal{C}$, such that $\sigma_{\mathcal{C}}(X) \subseteq G=$ $C-A \prec C$. So $X \subseteq Q-A$ which is a closed set. Then $\sigma_{\mathcal{C}}(X) \subseteq Q-A \subset Q$. 
5. $\Longrightarrow$ 1. We prove that $\mathcal{C}+\{Q\}$ is a closure system by showing that $(G \cap Q) \in \mathcal{C}+\{Q\}$ for any $G \in \mathcal{C}$. If $G$ and $Q$ are comparable the result holds. Otherwise we have $G \cap Q \subset Q$ and so $\sigma_{\mathcal{C}}(G \cap Q) \subset$ $\sigma_{\mathcal{C}}(Q)=C$ (indeed $\sigma_{\mathcal{C}}(G \cap Q)=\sigma_{\mathcal{C}}(Q)$ would imply $Q \subset \sigma_{\mathcal{C}}(Q)=\sigma_{\mathcal{C}}(G \cap Q) \subseteq G$, a contradiction). Then by item 5 , $\sigma_{\mathcal{C}}(C \cap Q) \subset Q$. So $\sigma_{\mathcal{C}}(C \cap Q) \subseteq C \cap Q$, which means $C \cap Q=\sigma_{\mathcal{C}}(C \cap Q) \in \mathcal{C}$.

Remark: In [22] Guigues and Duquenne defined a saturated gap as a set $Q$ satisfying item [5] above. In [20] Ganter proved the equivalence between items 1] and 5] (see also Wild [39] and Caspard and Monjardet [9]).

Lemma 2 Let $C$ be a closure system on $S, \sigma_{\mathcal{C}}$ the associated closure operator and $Q$ a subset of $S$.

1. If $Q$ is a $C$-quasi-closed set, then exC $\subseteq Q \subset C$ and, for every $x \in \operatorname{exC},(Q-x) \in C$.

2. If $Q \subset C=\sigma_{C}(Q)=\sigma_{C}(e x C)$, then $[Q$ is a $C$-quasi-closed set if and only if for every $x \in e x C$, $(Q-x) \in C$ ]. (In particular exC is a $C$-quasi-closed set if and only if for every $x \in \operatorname{exC}$, ex $(C-x) \in$ C).

\section{Proof:}

(1) Since $e x C$ is the intersection of the bases of $C$, we have exC $\subseteq Q \subset C$ and, if $x \in e x C$, then $C-x \in \mathcal{C}$ and so $(C-x) \cap Q=Q-x \in C$.

(2) By item 1 , we only have to prove the sufficient condition. Assume that $Q \subset C=\sigma_{\mathcal{C}}(Q)=\sigma_{\mathcal{C}}(\operatorname{exC})$. We prove that $Q$ satisfies Condition 3 of Proposition 11, i.e. that for every closed set $G$ such that $G \prec C, G \cap Q \in C$. It is sufficient to prove that a closed set $G$ is covered by $C$ (if and) only if $G=(C-x)$ with $x \in e x C$ (since then $G \cap Q=(C-x) \cap Q=Q-x \in \mathcal{C})$. If $G \prec C=\sigma_{\mathcal{C}}(e x C)$, exC $\nsubseteq G$. Then there exists $x \in e x C$ such that $x \notin G$. So $G \subseteq(C-x) \prec C$ and finally $G=C-x$.

Lemma 3 Let $C$ be a closure system on $S, C \in C$ and $x \in(C-e x C)$.

1. $(C-x)$ is a $C$-quasi-closed set if and only if [for every $G \in \mathcal{C}$ with $G \prec C$ and $x \in G$, then $x \in e x G$ ].

2. Let $C \in C$ such that $C$ is a minimal dependent closed set. Then $C-x$ is a $C$-quasi-closed set.

Proof:

1 By Proposition 1, item 3 and Lemma $1(C-x)$ is a $C$-quasi-closed set if and only if for every closed set $G$ such that $G \prec C, G \cap(C-x)=(G-x) \in C$, if and only if for every closed set $G$ such that $G \prec C, x \in e x G$.

2 If $x \in G$ with $G \prec C$, then $x \in \operatorname{ex} G=G$, since $G$ is free. Then by item 1 , $(C-x)$ is a $C$-quasi-closed set.

Remark: Since the closed sets of a closure system $C$ are all free if and only if $C=2^{S}$, item 2 of the above Lemma proves the previous assertion that any closure system (distinct from $2^{S}$ ) admits quasi-closed sets.

We now define a converse notion (in some sense) of the notion of quasi-closed set. 
Definition 3 Let $\mathcal{C}$ be a closure system on $S . C \in \mathcal{C}$ is a deletable closed set (w.r.t. $\mathcal{C})$ if $(C-C)$ is a closure system on $S$.

The following result is obvious.

Lemma 4 Let $C$ be a closure system on $S . C \in C$ is a deletable closed set (w.r.t. $C$ ) if and only if $C$ is a meet-irreducible element of the lattice $C$.

We denote by $\mathcal{M}$ the set of all closure systems defined on a set $S$. The poset $(\mathcal{M}, \subseteq)$ is a well studied lattice (see for instance Caspard and Monjardet [9]) and we recall some of its properties below:

Theorem 1 The lattice $\mathcal{M}$ of all closure systems defined on a set $S$ of cardinality $n$ satisfies the following properties:

1. Its greatest element is the Boolean algebra $2^{S}$ and its least element is the closure system $\{S\}$.

2. Its atoms are the $\left(2^{n}-1\right)$ closure systems $\mathcal{C}_{A}=\{A, S\}$, for $A \subset S$.

3. Its meet-irreducible elements are the $n\left(2^{n}-1\right)$ closure systems $C_{A, x}=\{X \subseteq S: A \nsubseteq X$ or $x \in X\}$, for $A \subseteq S-x$ and $x \in S$ (with upper covers $C_{A, x}^{+}=C_{A, x}+\{A\}$ ).

4. For all $\mathcal{C}, C^{\prime} \in \mathcal{M}, \mathcal{C} \wedge C^{\prime}=\mathcal{C} \cap C^{\prime}$ and $\mathcal{C} \vee C^{\prime}=\left\{C \cap C^{\prime}, C \in \mathcal{C}, C^{\prime} \in C^{\prime}\right\}$.

5 .

$$
\begin{aligned}
C \prec C^{\prime} & \Longleftrightarrow \quad \text { there exists a quasi-closed set } Q \text { (w.r.t. } C \text { ) such that } \\
& C^{\prime}=C+\{Q\} \\
& \Longleftrightarrow \quad \text { there exists a meet-irreducible element } Q \text { of } C^{\prime} \text { such that } \\
& C=C^{\prime}-\{Q\}
\end{aligned}
$$

6. $\left(C_{A} \downarrow \mathcal{C}_{B, x}\right.$ if and only if $\left.B \subseteq A \subseteq S-x\right)$ and $\left(C_{A} \uparrow C_{B, x}\right.$ if and only if $\mathcal{C}_{A} \uparrow \mathcal{C}_{B, x}$ if and only if $B=A \subseteq$ $S-x)$.

7. $C_{A} \delta C_{B}$ if and only if $A \subseteq B \subset S$ (so the dependence relation $\delta$ is isomorphic with the Boolean lattice $2^{S}$, without the greatest element).

8. The dependence relation $\beta$ is symmetric and strongly connected.

9. The lattice $\mathcal{M}$ is atomistic and lower bounded and, consequently, lower locally distributive, meetsemimodular, ranked and join-pseudo complemented \$

10. The rank of the closure system $\mathcal{C}$ in $\mathcal{M}$ is $r(\mathcal{C})=|\mathcal{C}|-1$ and the length of $\mathcal{M}$ is $2^{n}-1$.

Notation: In the following, we omit the bracketings in the set notation. Thus $\{a\}$ will be denoted by $a$ and $\{b, c\}$ by $b c$.

The set $\mathcal{M}_{\emptyset}$ of all the closure systems containing $\emptyset$ is a lattice. Indeed, it is clear that $\mathcal{M}_{\emptyset}$ is the sublattice $\left[\{\emptyset, S\}, 2^{S}\right]$ of $(\mathcal{M}, \subseteq, \cap, \vee)$. Then from Theorem 1 we easily obtain the following results on $\mathscr{M}_{\emptyset}$.

Corollary 1 1. The atoms of $\mathcal{M}_{\emptyset}$ are the $2^{n}-2$ closure systems $C_{A, \emptyset}=\{\emptyset, A, S\}$, for $\emptyset \subset A \subset S$.

$\S$ For the definition of these classes of lattices see for instance [9] or [19]. 
2. The meet-irreducible elements of $\mathcal{M}_{\emptyset}$ are the $n\left(2^{n-1}-1\right)$ closure systems $\mathcal{C}_{A, x}$ with $\emptyset \subset A \subset S-x$ and $x \in S$.

3.

$$
\begin{aligned}
C \prec C^{\prime} & \Longleftrightarrow \quad \text { there exists a quasi-closed set } Q \text { for } C \text { with } C^{\prime}=C+\{Q\} \\
& \Longleftrightarrow \quad \text { there exists } Q \in M_{C^{\prime}} \text { with } C=C^{\prime}-\{Q\} .
\end{aligned}
$$

4. $\mathcal{M}_{0}$ is atomistic and lower bounded. It is therefore lower locally distributive, join-semidistributive, join-pseudo complemented, meet-semimodular and ranked (with $r(C)=|C|-2$ ).

5. The dependence relation $\delta$ is isomorphic with the Boolean lattice $2^{S}$, without the greatest and the least element.

6. The dependence relation $\beta$ is symmetric and strongly connected.

Remark: Everything that has been said in this section can directly be dualized to the union stable set families which contain $\emptyset$. Let us call these set families dual closure systems and denote by $\mathcal{U}$ the set of these families. $(\mathcal{U}, \subseteq)$ is obviously a lattice dual to $(\mathcal{M}, \subseteq)$. Moreover, if $\mathcal{C}$ is a dual closure system, a dual notion of a quasi-closed set for a closure system would be the notion of dual quasi-closed set for $\mathcal{C}$ defined as a set $R \subset S$ satisfying $R \notin C$ and such that $C+R$ is a dual closure system. This notion of dual quasi-closed set will be more generally applied to all $\cup$-stable systems.

In the following sections we study some particular closure systems, their lattices and their covering relations. Section 3 deals with $\cup$-closure systems and Section 4 with convex geometries.

\section{The lattice $\mathcal{M}^{\cup}$ of $\cup$-closure systems and its covering relation}

Definition 4 Let $S$ be a finite set. $A \cup$-closure system $C$ on $S$ is a closure system on $S$ that is stable under union: $C, C^{\prime} \in \mathcal{C}$ implies $\left(C \cup C^{\prime}\right) \in C$.

These $\cup$-closure systems are exactly the sublattices of the lattice $\left(2^{S}, \subseteq\right)$ of all subsets of $S$, which contain $S$. Since $\left(2^{S}, \subseteq\right)$ is distributive, so are all $\cup$-closure systems. The join- and meet-irreducible elements of a $\cup$-closure system $C$ will be respectively called the $\cup$-irreducibles and the $\cap$-irreducibles of $\mathcal{C}$ and we denote by $\mathcal{M}^{\cup}$ the set of all $\cup$-closure systems on $S$.

\section{Example:}

1. Recall that $\mathcal{C}_{A, B}=\{X \subseteq S: A \nsubseteq X$ or $B \subseteq X\}$. It is easy to check that

$$
\mathcal{C}_{A, B} \in \mathcal{M}^{\cup} \Longleftrightarrow[|A|=1 \text { or }(A=\emptyset \text { and }|B|=1)]
$$

2. A $\cup$-closure system containing $\emptyset$ is called a topology.

3. The $\cup$-closure systems $C(A)=\mathcal{C} \vee C_{A}$ (for any $\cup$-closure system $C$ and any $A \subset S$ ) will play an important role in this section. 
Lemma 5 Let $C \in \mathcal{C} \in \mathcal{M}^{\cup}$ and denote by $C_{1}=C-A_{1}, \ldots, C_{k}=C-A_{k}$ the $k$ lower covers of $C$ in $C$. $B \subseteq S$ is a basis of $C$ if and only if $B$ is a system of distinct representatives $(S D R)$ of $\left\{A_{1}, \ldots, A_{k}\right\}$, i.e. $B=\left\{a_{1}, \ldots, a_{k}\right\}$ with $a_{i} \in A_{i}$, for every $i=1, \ldots, k$.

Proof: First observe that $A_{i} \cap A_{j}=\emptyset$ for all $i \neq j$. Indeed if there exists $i \neq j$ such that $A_{i} \cap A_{j}=X \supset \emptyset$ then, since $C$ is $\cup$-stable, $C_{i} \subset C_{i} \cup C_{j}=C-X \subset C$, which is a contradiction.

$\Longrightarrow$ : Let $B$ be a basis of $C$. So for every $i=1, \ldots, k, B \cap A_{i} \neq \emptyset$ (if not $B \subset C_{i}$, which implies $\sigma(B) \subseteq C_{i}$, a contradiction). Assume that $B \cap A_{i} \supseteq\{x, y\}$ for some $i \leq k$ (i.e. $\left|B \cap A_{i}\right|>1$ ). Then, since $y \in B-x$ and $y \notin C_{i}, \sigma(B-x) \nsubseteq C_{i}$. And $\sigma(B-x) \not \subset C_{j}$ for every $j \neq i$, since there exists $a_{j} \in(B-x) \cap A_{j}$ with $a_{j} \notin C_{j}$. So $\sigma(B-x)=C$, a contradiction with the fact that $B$ is a basis. Finally $\left|B \cap A_{i}\right|=1$ for every $i=1, \ldots, k$.

$\Longleftarrow$ : Take any $S D R B=\left\{a_{1} \ldots a_{k}\right\}$ of $\left\{A_{1}, \ldots, A_{k}\right\}$. We have $\sigma(B) \subseteq C$. Assume that $\sigma(B) \subset C$. Then there exists $C_{i} \prec C$ such that $\sigma(B) \subseteq C_{i}$. But $a_{i} \in B-C_{i}$, a contradiction. So $\sigma(B)=C$. Let us now consider $B-a_{i}$ for some $i \leq k$. Then $B-a_{i} \subseteq C_{i}$ and so $\sigma\left(B-a_{i}\right) \subseteq C_{i} \subset C$.

Lemma 6 Let $Q$ be a $C$-quasi-closed set for $\mathcal{C} \in \mathcal{M}^{\cup}$. Then for all lower covers $C_{i}, C_{j}$ of $C$ in $\mathcal{C}, C_{i} \cap Q=$ $C_{j} \cap Q$.

Proof: Assume that there exists two lower covers $C_{i}$ and $C_{j}$ of $C$ satisfying $C_{i} \cap Q \neq C_{j} \cap Q$. Since $Q$ is quasi-closed, $C_{i} \cap Q$ and $C_{j} \cap Q$ belong to $C$ which is $\cup$-stable, so $\left(C_{i} \cap Q\right) \cup\left(C_{j} \cap Q\right) \in \mathcal{C}$. But $\left(C_{i} \cap Q\right) \cup\left(C_{j} \cap Q\right)=\left(C_{i} \cup C_{j}\right) \cap Q=C \cap Q=Q \notin \mathcal{C}$, a contradiction.

Proposition 2 Let $C$ be a $\cup$-closure system and $Q \subseteq S$ such that $\sigma(Q)=C \neq \emptyset$. The following are equivalent:

1. $Q$ is a $C$-quasi-closed set of $\mathcal{C}$,

2. $C \in J_{\mathcal{C}}$ and $C^{-} \cap Q \in \mathcal{C}$.

\section{Proof:}

11. 2. Since $\sigma(Q)=C, Q$ contains a basis of $C$. Let $C_{1}=C-A_{1}, \ldots, C_{k}=C-A_{k}$ be the $k$ lower covers of $C$ in $C$ and assume $k \geq 2$. By Lemma 5, there exists a $S D R B=\left\{a_{1}, \ldots, a_{k}\right\}$ of $\left\{A_{1}, \ldots, A_{k}\right\}$ such that $B \subseteq Q$. For all $i \neq j, a_{j} \in C_{i} \cap Q$ and $a_{j} \notin C_{j} \cap Q$. Then $C_{i} \cap Q \neq C_{j} \cap Q$, a contradiction with Lemma 6 So $C \in J_{\mathcal{C}}$ and, by Lemma 2 , item $3, C^{-} \cap Q \in \mathcal{C}$.

2. $\Longrightarrow$ 1. Immediate from Lemma2, item 3 .

The set $\mathcal{M}^{\cup}$ is obviously stable under intersection but it is not stable under the join operation of $\mathcal{M}$ (for instance, in $\mathcal{M}, \mathcal{C}_{a}=\{a, S\}$ and $\mathcal{C}_{b}=\{b, S\}$ (with $a \neq b$ ) are $\cup$-closure systems but their join in $\mathcal{M}$, $\mathcal{C}_{a} \vee \mathcal{C}_{b}=\{\emptyset, a, b, S\}$ is not $\cup$-stable). So $\mathcal{M}^{\cup}$ is an $\cap$-subsemilattice of $\mathcal{M}$. Since it contains $2^{S}$, it is a lattice and if we denote by $\mathcal{C}^{\cup}$ the $\cup$-closure of a closure system $\mathcal{C}$, i.e. $\mathcal{C}^{\cup}=\{\cup \mathcal{G}: \mathcal{G} \subseteq \mathcal{C}\}$ then the join operation in $\mathcal{M}^{\cup}$, denoted by $\vee_{\mathcal{M}^{\cup}}$, is given below: 
Lemma 7 Let $\mathcal{C}$ and $\mathcal{C}^{\prime}$ be two $\cup$-closure systems. $\mathcal{C} \vee \mathcal{M}^{\cup} \mathcal{C}^{\prime}=\left(\mathcal{C} \vee \mathcal{C}^{\prime}\right)^{\cup}=\left(\left\{C \cap C^{\prime}, C \in \mathcal{C}, C^{\prime} \in \mathcal{C}^{\prime}\right\}\right)^{\cup}$.

Notation: Throughout this section, the join operation in $\mathcal{M}^{\cup}$ will simply be denoted by $\vee$. We write $J^{\cup}$ and $M^{\cup}$ respectively the sets $J_{\mathcal{M}} \cup$ and $M_{\mathcal{M}^{\cup}}$. In order to give the characterizations of the order and covering relations on $\mathcal{M}^{\cup}$, we define, for $\mathcal{C} \in \mathcal{M}^{\cup}, I_{\mathcal{C}}=\left\{[X, Y] \subset \mathcal{C}: X \in J_{\mathcal{C}} \cup\{\min \mathcal{C}\}, Y \in M_{\mathcal{C}}\right.$ and $X \subseteq Y\}$.

Proposition 3 Let $C^{\prime}$ be a $\cup$-closure system.

$$
\left(\mathcal{C} \subseteq \mathcal{C}^{\prime} \text { and } \mathcal{C} \in \mathcal{M}^{\cup}\right) \quad \Longleftrightarrow \quad \exists I \subseteq I_{\mathcal{C}^{\prime}} \text { such that } \mathcal{C}=\mathcal{C}^{\prime}-\bigcup_{\left[X_{i}, Y_{i}\right]_{\mathcal{C}^{\prime}} \in I}\left[X_{i}, Y_{i}\right]_{\mathcal{C}^{\prime}}
$$

Moreover if $X_{i}=X$ for any $i$ such that $\left[X_{i}, Y_{i}\right]_{C^{\prime}} \in I$ then $X$ is a quasi-closed set for $C$.

Proof:

$\Longrightarrow$ : We must prove that for any $C \in C^{\prime}-\mathcal{C}$, there exists $[X, Y]_{C^{\prime}} \in I_{C^{\prime}}$ such that $C \in[X, Y]_{C^{\prime}} \subseteq C^{\prime}-\mathcal{C}$. Suppose that this does not hold for some $C_{0} \in C^{\prime}-\mathcal{C}$. We set $J_{0}=\left\{T \in J_{\mathcal{C}^{\prime}}: T \subseteq C_{0}\right\} \cup\left\{\min C^{\prime}\right\}$ and $M_{0}=\left\{U \in M_{C^{\prime}}: C_{0} \subseteq U\right\}$. Clearly, these two sets are non-empty and we have $\bigcup J_{0}=C_{0}=$ $\bigcap M_{0}$. Consequently, for all $T \in J_{0}$ and $U \in M_{0}$, there exists $Z_{T, U} \in \mathcal{C} \cap[T, U]_{\mathcal{C}^{\prime}}$ (if not, $C_{0} \in$ $[T, U]_{C^{\prime}} \subseteq C^{\prime}-\mathcal{C}$, a contradiction). We have $\bigcup_{T \in J_{0}} \bigcap_{U \in M_{0}} Z_{T, U} \in \mathcal{C}$. On the other hand, $C_{0}=\bigcup J_{0} \subseteq$ $\bigcup_{T \in J_{0}} \bigcap_{U \in M_{0}} Z_{T, U} \subseteq \bigcap_{M_{0}}=C_{0}$, that is $\bigcup_{T \in J_{0}} \bigcap_{U \in M_{0}} Z_{T, U}=C_{0} \in \mathcal{C}$, which is a contradiction.

$\Longleftarrow: \mathcal{C} \subseteq \mathcal{C}^{\prime}$ is obvious. Now suppose that $\mathcal{C}$ is not $\cup$-stable, i.e. there exists $X_{1}$ and $X_{2} \in \mathcal{C}$ with $X_{1} \cup X_{2} \in C^{\prime}-\mathcal{C}$ and so $X_{1} \cup X_{2} \neq \min C^{\prime}$. This implies that there exists $X \in J_{C^{\prime}}$ and $Y \in M_{C^{\prime}}$ with $X \subseteq Y$ and $X_{1} \cup X_{2} \in[X, Y]_{C^{\prime}}$. Since $C^{\prime}$ is a distributive lattice, $X \subseteq X_{1} \cup X_{2}$ implies $X \subseteq X_{1}$ or $X \subseteq X_{2}$ and so $X_{1} \nsubseteq Y$ or $X_{2} \nsubseteq Y$ (otherwise $X_{1}$ or $X_{2} \notin \mathcal{C}$ ) and finally $X_{1} \cup X_{2} \nsubseteq Y$, a contradiction. By duality, we would similarly prove that $\mathcal{C}$ is $\cap$-stable. Since $\mathcal{C}$ clearly contains $S, \mathcal{C}$ is a $\cup$-closure system contained in $C^{\prime}$.

At last if all $X_{i}$ are equal to $X$ then $C^{\prime} \cap X \subseteq X$ for any $C^{\prime} \in(C+\{X\})$ and, since $C^{\prime} \cap X \in \mathcal{C}, X$ is a quasi-closed set for $\mathcal{C}$.

\section{Remark:}

1. In this proposition, if $Y_{i}=Y$ for any $i$ such that $\left[X_{i}, Y_{i}\right]_{\mathcal{C}^{\prime}} \in I$, then $Y$ is a dual quasi-closed set for $C$.

2. In [36] Rival characterizes the sublattices of a distributive lattice. In terms of closure systems this amounts to characterize all $T_{0}$-topologies (see Definition 7, Section 4) contained in a $T_{0}$-topology. Then Proposition 3 is a generalization of Rival's result to arbitrary $\cup$-closure systems.

We define an order relation on the set $I_{C}$ by $[X, Y]_{C} \leq\left[X^{\prime}, Y^{\prime}\right]_{\mathcal{C}}$ if $X^{\prime} \subseteq X$ and $Y \subseteq Y^{\prime}$. Recall that $\mathcal{C}_{Q}=\{Q, S\}$ for any $Q \subset S$ and that for $\mathcal{C} \in \mathcal{M}^{\cup}, \mathcal{C}(Q)$ is the $\cup$-closure system $\mathcal{C}(Q)=\mathcal{C} \vee \mathcal{C}_{Q}$.

In Theorem 2 we will characterize the covering relation in the lattice $\mathcal{M}^{\cup}$ in terms of quasi-closed sets. We begin with a lemma. 
Lemma 8 Let $C$ be $a \cup$-closure system and $Q$ a quasi-closed set for $C$. Then the following holds:

$$
C(Q)=\mathcal{C}+\bigcup_{i=1 \ldots k}\left[Q, T_{i}\right]_{\mathcal{C}}(Q)
$$

with:

a) $T_{i} \in M_{\mathcal{C}(Q)}$ for any $i \leq k$

b) $T_{i} \| T_{j}$ for $i \neq j$

c) $(C(Q)-C) \cap J_{C(Q)}=Q$

Proof: $\mathcal{C}(Q)=\mathcal{C} \vee\{Q, S\}=\mathcal{C}+\{Q\}+\{C \cup Q: C \in \mathcal{C}$ and $C \cup Q \notin \mathcal{C}+\{Q\}\}$. Let $C_{1}, \ldots, C_{k}$ be the maximal elements of $\mathcal{C}$ such that $\left.\left(C_{i} \cup Q\right)=T_{i} \notin \mathcal{C}+\{Q\}\right\}$. Let $\left[Q, T_{i}\right]_{\mathcal{C}(Q)}=Q \cup\left\{R: Q \subseteq R \subseteq T_{i}, R=\right.$ $C \cup Q$ with $\left.C \subseteq C_{i}\right\} \subset(C(Q)-C)$ (indeed if $R=C_{i} \cup Q \in C$ then $R \cup C_{i}=Q \cup C_{i}=T_{i} \in \mathcal{C}$ ). Thus $\mathcal{C}(Q)=\mathcal{C}+\bigcup_{i=1 \ldots k}\left[Q, T_{i}\right]_{\mathcal{C}(Q)-\mathcal{C}}$

(b) If there exists $i \neq j$ such that, for example, $T_{i}=C_{i} \cup Q \subset C_{j} \cup Q=T_{j}$ then $C_{i} \cup C_{j} \cup Q=T_{j}$, which implies that $C_{i}$ and $C_{j}$ were not maximal.

(a) If there exists $i$ such that $T_{i}$ is the intersection of some elements of $C(Q)$ then these elements are not in any interval $\left[Q, T_{j}\right]_{\mathcal{C}(Q)}$ (since the $T_{i}$ 's are not comparable). So these elements belong to $\mathcal{C}$ and so does $T_{i}$, a contradiction.

(c) $Q \in J_{\mathcal{C}(Q)}$ since if $Q$ is obtained as the union of at least two elements of $\mathcal{C}(Q)$ (different from $Q$ ) then these elements belong to $C$ and so does $Q$, a contradiction. At last if $\left.R \in] Q, T_{i}\right]_{\mathcal{C}(Q)}, R=C_{i} \cup Q$ with $C_{i}, Q \in \mathcal{C}(Q)$ and so $R \notin J_{\mathcal{C}(Q)}$.

Definition $5 Q \subset S$ is a cover admissible quasi-closed set for $a \cup$-closure system $C$ if $Q$ is a quasi-closed set for $C$ satisfying $C \prec \mathcal{C}(Q)$.

Theorem 2 Let $\mathcal{C}$ and $C^{\prime}$ be two $\cup$-closure systems. The following assertions are equivalent.

1. $C \prec C^{\prime}$.

2. $C=C^{\prime}-[Q, T]_{\mathcal{C}^{\prime}}$ with $Q$ a cover admissible quasi-closed set for $C$ and $[Q, T]$ is $\leq$-minimal in $I_{C^{\prime}}$.

Proof:

1. $\Longrightarrow \mathcal{C} \prec \mathcal{C}^{\prime}$ implies $C \subseteq C^{\prime}$ and so, by Proposition 3, $C=C^{\prime}-\bigcup_{i=1 \ldots k}\left[X_{i}, Y_{i}\right]_{\mathcal{C}^{\prime}}$. If $k>1$ then $C^{\prime \prime}=C^{\prime}-\left[X_{1}, Y_{1}\right]_{\mathcal{C}^{\prime}}$ is a $\cup$-closure system which satisfies $C \subset C^{\prime \prime} \subset C^{\prime}$, a contradiction with $\mathcal{C} \prec$ $C^{\prime}$. Now suppose $k=1$ and $C=C^{\prime}-[X, Y]_{C^{\prime}}$ with $[X, Y]_{C^{\prime}}$ non $\leq$-minimal in $I_{C^{\prime}}$. Consider $\left[X^{\prime}, Y^{\prime}\right]_{\mathcal{C}^{\prime}} \in \min _{\mathcal{C}^{\prime}}$ with $\left[X^{\prime}, Y^{\prime}\right]_{\mathcal{C}^{\prime}} \leq[X, Y]_{\mathcal{C}^{\prime}}$ then $C^{\prime \prime \prime}=C^{\prime}-\left[X^{\prime}, Y^{\prime}\right]_{\mathcal{C}^{\prime}}$ satisfies $C \subset \mathcal{C}^{\prime \prime \prime} \subset C^{\prime}$, a contradiction. Finally $\mathcal{C} \prec C^{\prime}$ implies $C=C^{\prime}-[X, Y]_{C^{\prime}}$ with $[X, Y]_{\mathcal{C}^{\prime}} \in \min _{\mathcal{C}^{\prime}}$ and, by Proposition 3 and Definition $5, X$ is a cover admissible quasi-closed set for $C$. 
2. $\Longrightarrow$ 1. Immediate from Proposition 3 .

\section{Remark:}

1. Note that the interval $\left[\min C^{\prime}, T\right]$ is $\leq$-minimal in $I_{C^{\prime}}$ if and only if $\min C^{\prime}=T \in M_{C^{\prime}}$. This is the only case where $C^{\prime}-\min C^{\prime}$ remains $\cup$-stable.

2. In [10] (see also [36]) the authors characterize the maximal sublattices $L^{\prime}$ of a distributive lattice $L$ : $L-L^{\prime}$ is either an interval $[j, m]$ with $j \in J_{L}$ and $m \in M_{L}$ with no element of $J_{L} \cup M_{L}$ in $] j, m$, or is a unique element which is either doubly irreducible or the least or the greatest element of $L$. In terms of closure systems, this amounts to characterize maximal $T_{0}$-topologies contained in a $T_{0}$-topology. Then Theorem 2 is a generalization of these results to arbitrary $\cup$-closure systems.

We give the corollary below as a direct consequence of Theorem 2 .

Corollary 2 Let $\mathcal{C}$ be a $\cup$-closure system and $Q \subseteq S$. Then $Q$ is a cover admissible quasi-closed set for $\mathcal{C}$ (i.e. $\mathcal{C} \prec \mathcal{C}^{\prime}=\mathcal{C}(Q)$ ) if and only if $\mathcal{C}(Q)=\mathcal{C}+[Q, T]_{C^{\prime}}$ with $Q$ quasi-closed set for $\mathcal{C}$ such that $R \in[Q, T]_{C^{\prime}} \cap M_{C^{\prime}}$ implies $R=T$.

Recall that $T$ is said to be a dual quasi-closed set for $\mathcal{C} \in \mathcal{M}^{\cup}$ if $C \cup T \in \mathcal{C}$ for every $C \in \mathcal{C}$.

Corollary 3 Let $\mathcal{C}$ be a $\cup$-closure system and $Q$ a quasi-closed set for $\mathcal{C}$. If $Q$ is either a maximal quasi-closed set or a dual quasi-closed set for $\mathcal{C}$, then it is cover admissible.

Proof: Let $Q$ be a maximal quasi-closed set for $\mathcal{C}$. So there exists $\mathcal{C}^{\prime} \in \mathcal{M}^{\cup}$ such that $\mathcal{C} \prec \mathcal{C}^{\prime} \subseteq \mathcal{C}(Q)=$ $\mathcal{C}+\bigcup_{i=1 \ldots k}\left[Q, T_{i}\right]_{\mathcal{C}(Q)}$ (by Lemma 8). By Theorem 2, $\mathcal{C} \prec \mathcal{C}^{\prime}$ implies that $\mathcal{C}^{\prime}=\mathcal{C}+\left[Q^{\prime}, T\right]=\mathcal{C}\left(Q^{\prime}\right)$ with $Q^{\prime}$ quasi-closed set for $\mathcal{C}$. So there exists an interval $\left[Q, T_{i}\right]$ in $\mathcal{C}(Q)$ which contains $Q^{\prime}$. But since $Q$ is a maximal quasi-closed set for $\mathcal{C}, Q=Q^{\prime}$ and so $\mathcal{C} \prec \mathcal{C}^{\prime}=\mathcal{C}\left(Q^{\prime}\right)=\mathcal{C}(Q)$.

In the case where $Q$ is simultaneously a quasi-closed and a dual quasi-closed set for $\mathcal{C}$, it is clear that $\mathcal{C}(Q)=\mathcal{C}+\{Q\}$.

\section{Remark:}

1. It should be possible to define a notion of a cover admissible dual quasi-closed set and then to get the dual result of Corollary 3 (a minimal dual quasi-closed set is cover admissible).

2. Let $\mathcal{C} \in \mathcal{M}^{\cup}$. The set of all cover admissible quasi-closed sets $Q$ for $\mathcal{C}$ can vary from the whole set $Q_{C}$ of all quasi-closed sets $Q$ for $C$ to the set $\max Q_{C}$ of the maximal quasi-closed sets for $C$, as it is shown in the examples below.

\section{Example:}

1. For any $\mathcal{C}_{A}=\{A, S\}$, the set of all the quasi-closed sets for $\mathcal{C}_{A}$ is equal to $\{B \subset S: B \supset A$ or $B \subset A\}$. So $\mathcal{C}_{A} \prec \mathcal{C}_{A}(B)=\mathcal{C}_{A}+\{B\}$, for any quasi-closed set $B$ for $\mathcal{C}_{A}$. In other words, every quasi-closed set for $C_{A}$ is cover admissible. 
2. For any $\mathcal{C}_{x, B}, C_{x, B}=[\emptyset, S-x] \cup[B+x, S]$ so that $\mathcal{C}_{x, B}^{c}=\bigcup_{y \in B}[x, S-y]$. Then it is easy to check that the set of all quasi-closed sets for $\mathcal{C}_{x, B}$ is equal to $\bigcup_{y \in B}[x, B-y+x]$. So the set of maximal quasi-closed sets for $\mathcal{C}_{x, B}$ is $\{B-y+x, y \in B\}$. It is easy to check that these maximal quasi-closed sets are the only cover admissible quasi-closed sets for $\mathcal{C}_{x, B}$.

In the following we consider two $U$-closure systems $C$ and $C^{\prime}$, and we denote by $\sigma$ (respectively, $\sigma^{\prime}$ ) their associated closure operators and by $e x$ (respectively, $e x^{\prime}$ ) the set of extreme elements w.r.t. $\sigma$ (respectively, $\sigma^{\prime}$ ). As well we set up $J_{C}=J, M_{C}=M$ and $J_{C^{\prime}}=J^{\prime}, M_{C^{\prime}}=M^{\prime}$.

Corollary 4 Let $C$ be $a \cup$-closure system, $Q$ a cover admissible $C$-quasi-closed set for $C$ and let $C^{\prime}$ denote the $\cup$-closure system $\mathcal{C}^{\prime}=\mathcal{C}(Q)=\mathcal{C}+[Q, T]_{\mathcal{C}^{\prime}}$. Then:

$$
\begin{aligned}
J^{\prime}= & \begin{cases}J+\{\min C & \text { if } \sigma(Q)=\min C \\
J+\{Q\}-\{C\} & \text { if } C^{-} \cup Q=C \\
J+\{Q\} & \text { otherwise. }\end{cases} \\
M^{\prime}= & M+\{T\}-\left\{H \in M \mid H \cup Q \in[Q, T]_{C^{\prime}} \text { and } H^{+} \cap(H \cup Q)=H\right\} .
\end{aligned}
$$

\section{Proof:}

(1) First, according to Theorem 2 $Q$ is always a join-irreducible element of $C^{\prime}$, except in the case where $\sigma(Q)=\min C$. In this case, $Q$ is the least element of $C^{\prime}$ and $\min C$ becomes a join-irreducible element of $C^{\prime}$. So $J^{\prime}=J+\{\min C\}$. Take now $H \in J$ and assume that $H \notin J^{\prime}$. Then there exists $G \in \mathcal{C}$ such that $G \cup Q \in[Q, T]_{\mathcal{C}^{\prime}}$ and $H=(G \cup Q) \cup H^{-}$. So $G$ is distinct from $H$ (otherwise $G \cup Q=H$ ). So $Q \subset H$, which implies $Q \subset \sigma(Q)=C \subseteq H$. Recall that, by Proposition 2, $C \in J$. First assume $C \subset H$. Then $Q \subset C \subseteq H^{-}$and $H=G \cup Q \cup H^{-}=G \cup H^{-}$, a contradiction with $H \in J$. Assume now that $C=H$ and so $C=G \cup Q \cup C^{-}$. So $C^{-} \subseteq G \cup C^{-} \subset C$ implies $C^{-}=G \cup C^{-}$, hence $G \subseteq C^{-}$, i.e. $C=Q \cup C^{-}$, and $J^{\prime}=J+\{Q\}-\{C\}$. In the other cases, any $H \in J$ remains an element of $J^{\prime}$ and so $J^{\prime}=J+\{Q\}$.

(2) By Theorem 2, $T$ is the unique meet-irreducible element of $C^{\prime}-C$. Take now $H \in M$ and assume that $H \notin M^{\prime}$. Then there exists $G \in \mathcal{C}$ such that $G \cup Q \in[Q, T]_{C^{\prime}}$ and $H^{+} \cap(G \cup Q)=H$. So $H \subset G \cup Q$ and $H \subseteq H \cup Q \subseteq G \cup Q$. Then $H^{+} \cap(H \cup Q)=H$ and $H \cup Q \in C^{\prime}-C=[Q, T]_{C^{\prime}}$. Note, in particular, that if $\min C \in M$ then $\left(\min C \in M^{\prime}\right.$ if and only if $\min C \subset Q$ ).

We now aim to characterize the irreducible elements of the lattice $\mathcal{M}^{\cup}$. The case of the join-irreducible elements is obvious and is given without proof in the proposition below.

Proposition $4 J^{\cup}=\left\{C_{A}: A \subset S\right\}=J_{\mathscr{M}}$ and the lattice $\mathcal{M}^{\cup}$ is atomistic.

The following lemma presents without proof some results that will lead to some $\cap$-representations for a $\cup$-closure system and to a characterization of the $\cap$-irreducibles of $\mathcal{M}^{\cup}$. We recall that for all $x \in S$ and $B \subseteq S-x$, we have $\mathcal{C}_{x, B}=\{X \subseteq S: x \notin X$ or $B \subseteq X\}$ and $\mathcal{C}_{0, x}=\{X \subseteq S: x \in X\}$. We denote by $\mathcal{G}_{\mathcal{M} \cup}$ the set $\left\{C_{x, B}: x \in S, B \subseteq S-x\right\} \cup\left\{C_{\emptyset, x}: x \in S\right\}$.

Lemma 9 1. $\mathcal{C}_{x, B}$ and $C_{\emptyset, x}$ are $\cup$-closure systems. 
2. a. $C_{x, B}^{c}=\bigcup_{b \in B}[x, S-y]$.

b. $\mathcal{C}_{x, B} \subseteq \mathcal{C}_{z, B^{\prime}} \Longleftrightarrow\left(x=z\right.$ and $\left.B^{\prime} \subseteq B\right)$.

c. $C_{x, B}=\bigcap_{y \in B} C_{x, y}$.

3. a. $\mathcal{C}_{\emptyset, x}^{c}=[\emptyset, S-x]$.

b. $\mathcal{C}_{0, x} \subseteq \mathcal{C}_{\emptyset, z} \Longleftrightarrow x=z$.

c. $C_{\emptyset, x} \subseteq C_{z, t} \Longleftrightarrow x=t$.

d. $\mathcal{C}_{z, t} \nsubseteq \mathcal{C}_{\emptyset, x}$.

4. Let $\mathcal{C}$ be an element of $\mathcal{G}_{\mathcal{M}} \cup$ and $\mathcal{C}^{\prime} a \cup$-closure system. Then:

$$
C^{\prime} \subseteq \mathcal{C} \Longleftrightarrow\left\{\begin{array}{l}
B \subseteq\left(\phi_{C^{\prime}}(x)-x\right) \text { if } \mathcal{C}=C_{x, B} \\
x \in \phi_{\mathcal{C}^{\prime}}(\emptyset) \text { if } \mathcal{C}=\mathcal{C}_{\emptyset, x}
\end{array}\right.
$$

According to this lemma, we can define some $\cap$-representations of a $\cup$-closure system by means of the elements of $\mathcal{G}_{\mathcal{M}} \cup$.

Proposition 5 Let $\mathcal{C}$ be a $\cup$-closure system and $\sigma=\sigma_{\mathcal{C}}$ the associated closure operator.

$$
\begin{aligned}
C & =\bigcap\left(\left\{\mathcal{C}_{x, B} \in \mathcal{G}_{\mathcal{M}^{\cup}}: B \subseteq \sigma(x)-x\right\} \cup\left\{\mathcal{C}_{\emptyset, y} \in \mathcal{G}_{\mathcal{M}^{\cup}}: y \in \sigma(\emptyset)\right\}\right) \\
& =\bigcap\left(\left\{\mathcal{C}_{x, B} \in \mathcal{G}_{\mathcal{M}^{\cup}}: B=\sigma(x)-x\right\} \cup\left\{\mathcal{C}_{\emptyset, y} \in \mathcal{G}_{\mathcal{M}^{\cup}}: y \in \sigma(\emptyset)\right\}\right)
\end{aligned}
$$

Proof: We write $\mathcal{G}_{\mathcal{M}}^{\prime} \cup$ the set $\left\{\mathcal{C}_{x, B} \in \mathcal{G}_{\mathcal{M}} \cup: B \subseteq \sigma(x)-x\right\} \cup\left\{\mathcal{C}_{\emptyset, y} \in G_{\mathcal{M}}: y \in \phi(\emptyset)\right\}$. By the previous lemma, $\mathcal{C} \subseteq \cap \mathcal{G}_{\mathcal{M} \cup}^{\prime}$ is obvious. Now suppose there exists $X$ in $\cap \mathcal{G}_{\mathcal{M} \cup}^{\prime}$ such that $X \notin \mathcal{C}$, i.e. $X \subset \sigma(X)=\bigcup_{x \in X} \sigma(x)$. So there exists $z \in X$ such that $\{z\} \subset \sigma(z)$ and $\sigma(z)-z \nsubseteq X X$. By definition $\mathcal{C}_{z, \sigma(z)-z} \in \mathcal{G}_{\mathcal{M}^{\cup}}^{\prime}$ and we have $X \notin \mathcal{C}_{z, \sigma(z)-z}$, which is a contradiction.

The second equality is a direct consequence of item $2 \mathrm{~b}$ in Lemma 9

These $\cap$-representations of a $\cup$-closure system easily lead to a characterization of the $\cap$-irreducible elements of $\mathcal{M}^{\cup}$.

Corollary 5 The set $M^{\cup}$ of the $\cap$-irreducible elements of the lattice $\mathcal{M}^{\cup}$ is characterized as follows:

$$
\begin{aligned}
M^{\cup} & =\left\{\mathcal{C}_{x, y}: x, y \in S \text { and } x \neq y\right\} \cup\left\{\mathcal{C}_{0, x}: x \in S\right\} \\
& =\left\{\text { coatoms of } \mathcal{M}^{\cup}\right\} \cup\left\{\mathcal{C}_{\emptyset, x}: x \in S\right\} .
\end{aligned}
$$

Proof: Let $C$ be a $\cup$-closure system. If $C$ is an $\cap$-irreducible of $\mathcal{M}^{\cup}$, then by Proposition 5 , there exists $x \in S$ and $B \subseteq S-x$, such that $C=\mathcal{C}_{x, B}$ or $\mathcal{C}_{\emptyset, x}$. Suppose $C=\mathcal{C}_{x, B}$ with $|B|>1$. By item 2c in Lemma9. $C=\bigcap_{y \in B} C_{x, y}$, a contradiction, and so $B$ is a singleton. Let now $x$ and $z$ be two distinct elements of $S$. $\mathcal{C}_{x, z}$ contains all singletons $\{t\}$, for $t \neq x$. Since $\{x\}$ is quasi-closed for $\mathcal{C}_{x, z}$, it belongs to an upper cover $C^{\prime}$ of $\mathcal{C}_{x, z}$. Thus, $C^{\prime}$ contains all singletons $\{t\}$, for $t \in S$, which implies $C^{\prime}=2^{S}$, and $\mathcal{C}_{x, z}$ is a coatom of $\mathcal{M}^{\cup}$.

As for $C_{\emptyset, x}$, it has $\emptyset$ as unique quasi-closed set, so $C_{\emptyset, x}$ has a unique upper cover $C_{\emptyset, x}^{+}=C_{\emptyset, x}+\{\emptyset\}$, and $\mathcal{C}_{\emptyset, x}$ is $\cap$-irreducible. It is not a coatom since $\mathcal{C}_{\emptyset, x}^{+}$contains $\{x\}$ as unique singleton.

In order to describe the dependence relations $\delta$ and $\beta$ on the irreducible elements of $\mathcal{M}^{\cup}$, we first give the arrow relations on this lattice. The proofs are easy to check and are left to the reader. 
Lemma 10 Let $C_{A}$ be an element of $J^{\cup}$ and $C_{Y, y}$ an element of $M^{\cup}$ (i.e. $|Y| \leq 1$ ). Then the following holds:

$$
\begin{aligned}
\mathcal{C}_{A} \subseteq \mathcal{C}_{Y, y} & \Longleftrightarrow A \in \mathcal{C}_{Y, y} \\
& \Longleftrightarrow[y \in A \text { if } Y=\emptyset] \text { and }[(y \in A \text { and } x \notin A) \text { if } Y=\{x\}] \\
\mathcal{C}_{A} \downarrow \mathcal{C}_{Y, y} & \Longleftrightarrow \mathcal{C}_{A} \nsubseteq \mathcal{C}_{Y, y} \\
& \Longleftrightarrow[A \subseteq S-y \text { if } Y=\emptyset] \text { and }[(x \in A \text { and } y \notin A) \text { if } Y=\{x\}] \\
\mathcal{C}_{A} \uparrow \mathcal{C}_{Y, y} & \Longleftrightarrow \mathcal{C}_{A} \uparrow \mathcal{C}_{Y, y} \\
& \Longleftrightarrow[A=\emptyset \text { if and only if } Y=\emptyset] \text { and }[\{x\} \subseteq A \subseteq S-y \text { if } Y=\{x\}] .
\end{aligned}
$$

It is now possible to characterize the dependence relations $\delta$ and $\beta$ on the irreducible elements of $\mathcal{M}^{\cup}$.

Proposition 6 Let $\mathcal{C}_{A}$ and $\mathcal{C}_{B}$ be two elements of $J^{\cup}$.

1. If $A, B \neq \emptyset$ then:

$$
C_{A} \delta C_{B} \Longleftrightarrow \exists x, y \in S \text { such that }\{x\} \subseteq A \cap B \subseteq A \cup B \subseteq S-y
$$

2. For any $B \subset S$, we have $C_{\emptyset} \delta C_{B}$.

3. For $\emptyset \subset B \subset S$, we have $C_{B} \delta^{c} C_{\emptyset}$.

The relation $\delta$ is not strongly connected.

Proposition 7 Let $\mathcal{C}_{\emptyset, x}, \mathcal{C}_{\emptyset, y}, \mathcal{C}_{x, y}$ and $\mathcal{C}_{z, t}$ be meet-irreducible elements of $\mathcal{M}^{\cup}$. The following holds:

1. $\mathcal{C}_{\mathbb{D}, x} \beta \mathcal{C}_{\mathbb{D}, y}, \forall x, y \in S$.

2. $\mathcal{C}_{\emptyset, x} \beta C_{y, z} \Longleftrightarrow x \neq y$.

3. $\mathcal{C}_{x, y} \beta \mathcal{C}_{z, t} \Longleftrightarrow x \neq t$ and $y \neq z$.

The relation $\beta$ is strongly connected.

The theorem below summarizes some known results and adds new ones on the lattice $\mathcal{M}^{\cup}$ :

Theorem 3 The lattice $\left(\mathcal{M}^{\cup}, \subseteq\right)$ of all $\cup$-closure systems defined on a set $S$ is a $\cap$-subsemilattice of the lattice $\mathcal{M}$, which contains $2^{S}$ and which satisfies:

1. The join and meet operations of $\mathcal{M} \cup$ are:

a. $\mathcal{C} \vee C^{\prime}=\left\{C \cap C^{\prime}, C \in \mathcal{C}, C^{\prime} \in \mathcal{C}^{\prime}\right\}^{\cup}=\bigcup\left\{\mathcal{H} \subseteq\left\{C \cap C^{\prime}, C \in \mathcal{C}, C^{\prime} \in C^{\prime}\right\}\right\}$

b. $C \wedge C^{\prime}=C \cap C^{\prime}$

2. The join-irreducible and the meet-irreducible elements of $\mathcal{M}^{\cup}$ are characterized as follows:

a. $J^{\cup}=\left\{\mathcal{C}_{A}: A \subset S\right\}=J_{\mathscr{M}}\left(\right.$ so $\mathcal{M}^{\cup}$ is atomistic) 
b. $M^{\cup}=\left\{\mathcal{C}_{x, y}: x, y \in S\right.$ and $\left.x \neq y\right\} \cup\left\{\mathcal{C}_{\emptyset, x}: x \in S\right\}$

3. $\mathcal{C} \prec C^{\prime}$ if and only if $C^{\prime}=C(Q)=C+[Q, T]_{C^{\prime}}$ with $Q$ quasi-closed set for $C$ such that $(R \in$ $\left.[Q, T]_{\mathcal{C}^{\prime}} \cap M_{\mathcal{C}(Q)}\right)$ implies $R=T$.

4. For $|S| \geq 3$, the lattice $\mathcal{M}^{\cup}$ is neither coatomistic, nor ranked, nor complemented, nor join-pseudo complemented, nor meet-pseudo complemented.

Proof: The points (1), (2) and (3) have already been proved. For (4), first observe that in the case where $|S| \leq 2$, the lattice $\mathcal{M}^{\cup}$ is Boolean. Now for $|S| \geq 3, \mathcal{M}^{\cup}$ is neither coatomistic (since $\mathcal{C}_{\emptyset, x}$ is a meetirreducible contained in the coatom $C_{y, x}$ ), nor ranked. It is not complemented since, for instance, the $\cup$-closure system $\{\emptyset, S\}$ has no complement in $\mathcal{M}^{\cup}$ (more generally, $\mathcal{C}_{A}=\{A, S\}$ has a complement in $\mathcal{M}^{\cup}$ if and only if $A=S-x$ for some $x \in S$ ). $\mathcal{M}^{\cup}$ is not join-pseudo complemented. Indeed a lattice $L$ is join-pseudo complemented if and only if every coatom of $L$ has a join-pseudo complement (see [11]). The coatom $\mathcal{C}_{1,2}$ of $\mathcal{M}^{\cup}$ on $S=\{1,2,3\}$ has two minimal join-semi complements which are $\mathcal{C}_{13}$ and $\mathcal{C}_{1}$. At last, $\mathcal{M}^{\cup}$ is not meet pseudo-complemented. For instance, consider the $\cup$-closure system $\mathcal{C}_{1}=\{1, S\}$ on $S=\{1,2,3\} . \mathcal{C}_{1}$ has two maximal meet semi-complements, $\mathcal{C}=\{\emptyset, 2,3,12,23, S\}$ and $C^{\prime}=\{\emptyset, 2,3,13,23, S\}$.

\section{Remark:}

1. Obviously the set of closure operators associated with $\cup$-closure systems is a lattice dual of $\mathcal{M}^{\cup}$.

2. As already said, a $\cup$-closure system is a topology if it contains $\emptyset$. The set $\mathcal{T}$ of all topologies on $S$ is the interval $\left[\{\emptyset, S\}, 2^{S}\right]$ of the lattice $\mathcal{M}^{\cup}$ and so, it is a sublattice of $\mathcal{M}^{\cup}$. But since this lattice or the dual lattice of preorders have already been well studied in the finite (and infinite) case(s), we send the reader back to the references [2], [4], [18], [26], [28] and [37]. Just note that, unlike the lattice $\mathcal{M}^{\cup}, \mathcal{T}$ is a coatomistic and complemented lattice.

\section{The lattice $\mathcal{G}^{+}$of convex geometries and its covering relation}

Definition 6 A closure system on $S$ is a convex geometry if it satisfies the two following properties:

1. The empty set $\emptyset$ is closed.

2. For every closed set $C \neq S$ there exists $x \notin C$ such that $C+x$ is a closed set.

Using easy or well-known results (Edelman [16], Edelman and Jamison [15]) and the characterization of join-irreducible elements of an arbitrary closure system (re [34]) we can state the following:

Lemma 11 Let $\mathcal{C}$ be a convex geometry on $S$ and $(\mathcal{C}, \subseteq)$ the associated lattice. Then $(\mathcal{C}, \subseteq)$ is lower locally distributive and its covering relation is characterized by:

$$
\forall C, C^{\prime} \in \mathcal{C},\left(C^{\prime} \prec C \Longleftrightarrow \exists x \in e x C, C^{\prime}=C-x\right)
$$

Moreover, if $\sigma_{\mathcal{C}}$ denotes the closure operator associated with $\mathcal{C}$, the sets $\sigma_{\mathcal{C}}(x), x \in S$ are all distinct and are exactly the join-irreducible elements of $\mathcal{C}$. 


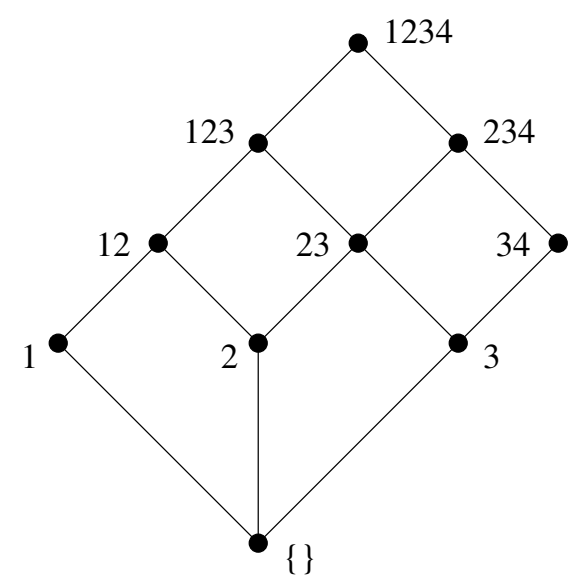

Fig. 1: The convex geometry $C=\{\emptyset, 1,2,3,12,23,34,123,234,1234\}$

Example: The closure system $C=\{\emptyset, 1,2,3,12,23,34,123,234,1234\}$ is a convex geometry (see Figure 11.

A particular class of convex geometries is the class of all $T_{0}$-topologies:

Definition 7 A $T_{0}$-topology on a set $S$ is a $\cup$-stable convex geometry. Equivalently it is a topology that is to say a $\cup$-closure system containing $\emptyset-C$ such that $x \neq y$ implies $\sigma_{\mathcal{C}}(x) \neq \sigma_{\mathcal{C}}(y)$. A linear topology is a $T_{0}$-topology $C$ such that there exists a linear order $L=x_{1}>\ldots>x_{i}>\ldots>x_{n}$ on $S$ with $\mathcal{C}=\left\{\left\{x_{i}, x_{i+1} \ldots, x_{n-1}, x_{n}\right\}, x_{i} \in S\right\}+\{\emptyset\}$.

There are many ways to define convex geometries and their corresponding closure operators (see for instance [24], [29] and [32]), that we partially list below:

Lemma 12 Let $C$ be a closure system containing $\emptyset$ and let $\sigma_{C}$ be the associated closure operator. The following properties are equivalent:

1. C is a convex geometry,

2. Every closed set of $C$ is the closure of its extreme elements,

3. Every closed set of $C$ has a unique basis, which is exC,

4. For every $X \subseteq S, \operatorname{exX}=\operatorname{ex\sigma _{C}}(X)$,

5. $\sigma_{\mathcal{C}}(\emptyset)=\emptyset$ and $\left[x, y \notin \sigma_{\mathcal{C}}(X), x \neq y\right.$ and $\left.y \in \sigma_{\mathcal{C}}(X+x)\right]$ imply $x \notin \sigma_{\mathcal{C}}(X+y)$.

Thus a convex geometry $\mathcal{C}$ induces a partition of $2^{S}$ into Boolean intervals $[e x C, C], C \in \mathcal{C}$, such that, for every $X \in[e x C, C], e x X=e x C$ and $\sigma_{C}(X)=C$.

The second condition of item 5 is called the antiexchange property. We will say that a closure operator is an antiexchange closure operator if it satisfies this condition. 
We denote by $\mathcal{G}$ the poset of all convex geometries defined on a set $S$, partially ordered by set inclusion. The join in $\mathcal{M}$ of two convex geometries is still a convex geometry (which is easy to check), but their intersection is not necessarily a convex geometry (for example with $|S|=2,\{\emptyset, 1,12\} \cap\{\emptyset, 2,12\}=$ $\{\emptyset, 12\} \notin \mathcal{G}) . \quad \mathcal{G}$ is therefore a join-subsemilattice of the lattice $\mathcal{M}$ that was studied in particular by Edelman and Jamison ([15] 1985). The following theorem gives some properties of this semilattice, but in order to simplify their statement we shall add a zero element $0_{\mathcal{G}}$ to $\mathcal{G}$ in order to obtain a lattice denoted by $\mathcal{G}^{+}$.

Theorem 4 The lattice $\mathcal{G}^{+}$of all convex geometries on a set $S$ of cardinality $n$ is a join-subsemilattice of the lattice $\mathcal{M}$ of all the closure systems on $S$, having the following properties:

1. Its greatest element is the Boolean algebra $2^{S}$.

2. Its join-irreducible elements are exactly the atoms and are equal to the $n$ ! linear topologies $\mathcal{L}$ defined on $S$.

3. Its meet-irreducible elements are the $n 2 n-1$ closure systems $\mathcal{C}_{A, x}$, for all $A \subseteq S$ and $x \notin A$ (with $\left.C_{A, x}^{+}=C_{A, x}+\{A\}\right)$.

4. For all $\mathcal{C}, C^{\prime} \in \mathcal{G}, \mathcal{C} \vee C^{\prime}=\left\{C \cap C^{\prime}, C \in \mathcal{C}, C^{\prime} \in \mathcal{C}^{\prime}\right\}$.

5. For all $\mathcal{C}, \mathcal{C}^{\prime} \in \mathcal{G}$,

$$
C \wedge C^{\prime}=\left\{\begin{array}{cl}
0_{\mathcal{G}} & \text { if } C \cap C^{\prime} \text { contains no linear topology } \\
\vee\left\{\mathcal{L} \mid \mathcal{L} \text { linear topology contained in } C \cap C^{\prime}\right\} & \text { otherwise. }
\end{array}\right.
$$

6. $\mathcal{G}^{+}$is atomistic and ranked.

7. The rank of the convex geometry $\mathcal{C}$ is $r(\mathcal{C})=|\mathcal{C}|-n$ (so $\mathcal{C} \prec \mathcal{C}^{\prime}$ implies $\left|\mathcal{C}^{\prime}\right|=|\mathcal{C}|+1$ ), and the minimum cardinality of a set $€$ of linear topologies with $C=\bigvee$ $C$ equals the width of the poset $M_{C}$.

8. The length of $\mathcal{G}^{+}$is $2^{n}-n$.

9. For $n \geq 3, \mathcal{G}^{+}$is neither upper nor lower semimodular.

Proof: The proofs of Properties 1, 2, 4, 5, 6,7 and 8 can be found in Edelman and Jamison ([15] 1985) or Edelman and Saks ([17] 1988). Just note that our Corollary 7 is the result proved in [15] in order to show that $\mathcal{G}^{+}$is ranked. Property 9 can easily be checked. We finally have to prove the characterization of the meet-irreducible elements of $\mathcal{G}^{+}$given in Property 3 In Theorem 1 it has been recalled that the closure systems $C_{A, x}=\{X \subseteq S: A \nsubseteq X$ or $x \in X\}$, for $A \subseteq S-x$, are the meet-irreducible elements of the lattice $\mathcal{M}$ of all the closure systems on $\mathrm{S}$. It is therefore sufficient to show that such a closure system is a convex geometry. Assume that there exists $C_{A, x}$ which is not a convex geometry. Then there exists $C \in C_{A, x}$ such that for every $y \notin C, C+y \notin C_{A, x}$, i.e. $A \subseteq C+y \subseteq S-x$. Since $C=\bigcap\{C+y, y \notin C\}$, we get $A \subseteq C \subseteq S-x$, a contradiction.

Our aim is to characterize the covering relation $\prec$ in $\mathcal{G}^{+}$. To do so we introduce the notions of $\mathcal{G}$ admissible $C$-quasi-closed set and of $\mathcal{G}$-deletable closed set. 


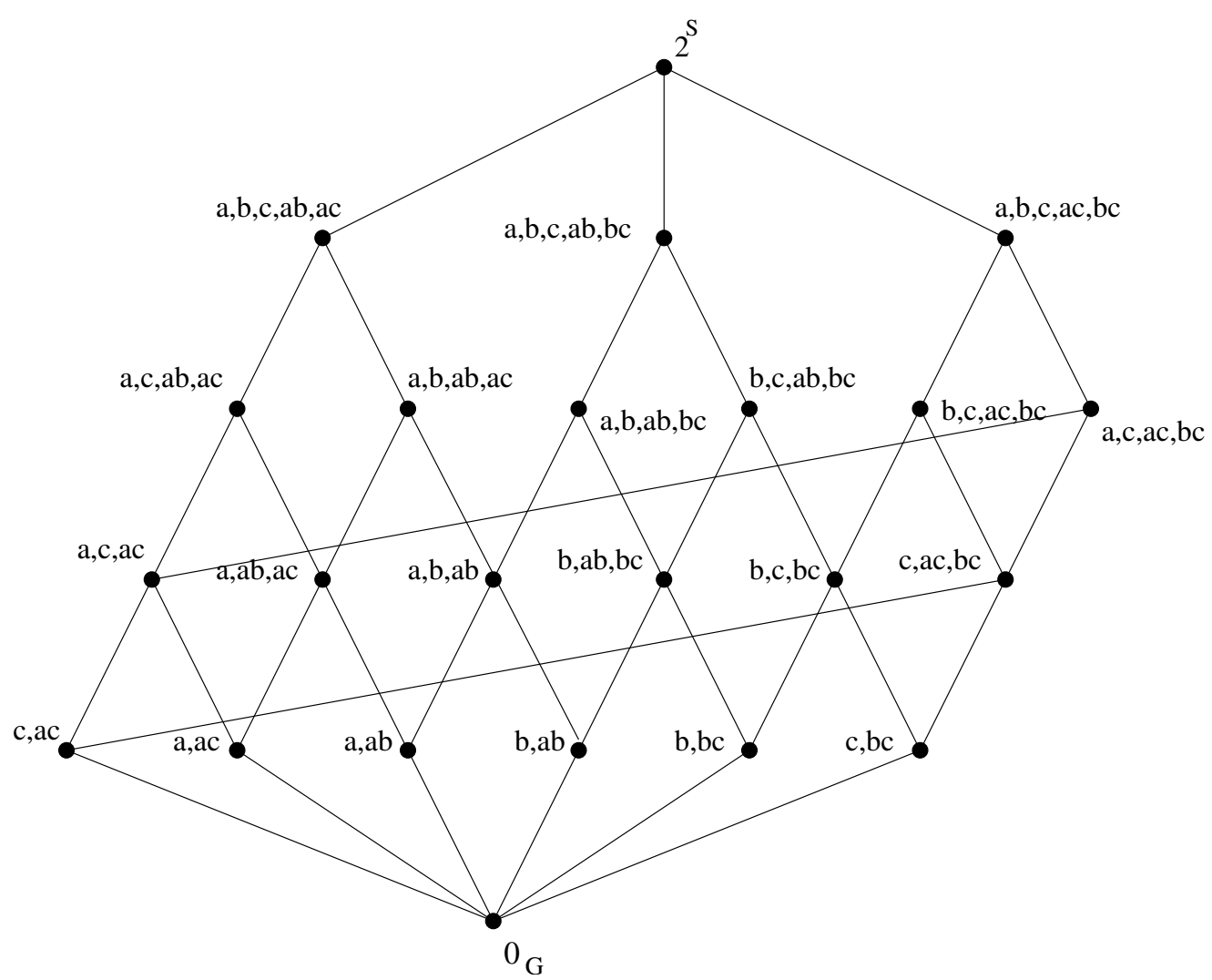

Fig. 2: The lattice $\mathcal{G}^{+}$of the convex geometries on a set $S=\{a, b, c\}$ (here a convex geometry is denoted by its elements different from $\emptyset$ and $S$ ).

Definition 8 Let $C$ be a convex geometry on $S$. A subset $Q$ of $S$ is a $\mathcal{G}$-admissible quasi-closed set (w.r.t. C) if $Q \notin C$ and $C+\{Q\}$ is a convex geometry. If $\sigma_{\mathcal{C}}(Q)=C$, we shall say that $Q$ is a $\mathcal{G}$-admissible $C$-quasi-closed set.

Definition 9 A closed set $C$ of a convex geometry $C$ is a $\mathcal{G}$-deletable closed set of $\mathcal{C}$ if $\mathcal{C}-\{C\}$ is a convex geometry.

We have seen that, in $\mathcal{G}^{+}$, the fact that $\mathcal{C} \prec \mathcal{C}^{\prime}$ implies $\left|\mathcal{C}^{\prime}\right|=|\mathcal{C}|+1$ (Theorem 44). This shows that $\mathcal{G}$-admissible quasi-closed sets always exist. So the following holds:

Fact 1 Let $C$ and $C^{\prime}$ be two convex geometries. The three assertions below are equivalent:

1. $C \prec C^{\prime}$,

2. There exists a $\mathcal{G}$-admissible quasi-closed set $Q$ (w.r.t. $C$ ) such that $C^{\prime}=\mathcal{C}+\{Q\}$,

3. There exists a $\mathcal{G}$-deletable closed set $Q$ of $C^{\prime}$ such that $C=C^{\prime}-\{Q\}$. 
Before characterizing the $\mathcal{G}$-admissible quasi-closed sets, we note that an immediate application of Lemmas 2 and 3 (item 2) allows us to characterize the $C$-quasi-closed sets of a convex geometry $C$, i.e. the sets $Q$ such that $C+\{Q\}$ is a closure system with $Q \subset \sigma_{C}(Q)=C$ :

Lemma 13 Let $C$ be a convex geometry on $S$ and $Q \subset S$. The two following properties are equivalent:

1. There exists a closed set $C$ of $C$ such that $Q$ is a $C$-quasi-closed set,

2. exC $\subseteq Q \subset C$ and for every $u \in \operatorname{exC},(Q-u) \in \mathcal{C}$.

We can now characterize the $\mathcal{G}$-admissible quasi-closed sets of a convex geometry.

Proposition 8 Let $\mathcal{C}$ be a convex geometry on $S, \sigma$ the associated antiexchange closure operator and $Q \subset S$. The following assertions are equivalent:

1. $Q$ is a $\mathcal{G}$-admissible quasi-closed set (i.e. $\mathcal{C} \prec \mathcal{C}+\{Q\} \in \mathcal{G}$ ),

2. There exists a closed set $C$ of $C$ and $u \in C$ such that $Q=C-u$ is a $C$-quasi-closed set for $\mathcal{C}$,

3. There exists a closed set $C$ of $C$ and $u \in C-$ exC such that exC $\subseteq Q=C-u$ and for every $t \in$ exC, $Q-t \in \mathcal{C}$,

4. There exists a closed set $C$ of $C$ and $u \in C-$ exC such that exC $\subseteq Q=C-u$ and for every closed set $G$ of $C$ such that $G \prec C, u \in \operatorname{ex} G$,

5. There exists a closed set $C$ of $C$ and $u \in C-$ exC such that exC $\subseteq Q=C-u$ and for every $X$ such that $u \in X \subseteq \sigma(X) \subset C$, then $u \in \operatorname{exX}$.

\section{Proof:}

1. $\Longrightarrow$ 2. By hypothesis, $Q \notin C$ and $C+\{Q\}$ is a convex geometry. Then by Lemma 11 there exists $C \in \mathcal{C}$ such that $Q+u=C$. If $\sigma(Q) \neq C$ then $Q \in \mathcal{C}$ which is impossible. Then $\sigma(Q)=C$ and $Q=C-u$ is a $C$-quasi-closed set.

2. 3. By applying Lemma2 item 1 with $Q=C-u$ we get $e x C \subseteq Q=C-u$ and for every $t \in e x C$, $(Q-t) \in \mathcal{C}$.

3. $\Longrightarrow$ 4. For $G \in \mathcal{C}, G \prec C$ is satisfied if and only if there exists $t \in e x C$ such that $G=C-t$ (Lemma 11. Then $u \in G$ (since $u \in C-e x C \subseteq C-t$ ) and $G-u=C-\{u, t\}=Q-t \in \mathcal{C}$, i.e. $u \in$ ex $G$.

4. $\Longrightarrow$ 5. Let $X \subseteq S$ be such that $X \subseteq \sigma(X) \subset C$, with $u \in X$. Then there exists a closed set $H$ such that $\sigma(X) \subseteq H \prec C$. So $X-u \subset X \subseteq \sigma(X)$ and $X-u \subset H-u$ imply $X-u \subseteq \sigma(X) \cap(H-u)$ and so $X-u \subseteq \sigma(X-u) \subseteq \sigma(X) \cap(H-u)=\sigma(X)-u \subset \sigma(X)$. This implies $\sigma(X-u) \subset \sigma(X)$, i.e. $u \in \operatorname{exX}$. 
5. $\Longrightarrow$ 1. We first show that $Q=C-u$ is a $C$-quasi-closed set. First $u \in(C-e x C)$ implies $\sigma_{C}(Q)=C$. By Lemmas 2 and 3 (item 2 ) we have to prove that for every $t \in \operatorname{exC},(Q-t)=C-\{u, t\} \in \mathcal{C}$. But $t \in e x C$ implies $C-t \in C$ and $(C-t) \prec C$. According to the assertion 5, $u \in e x(C-t)$ and then $C-\{u, t\} \in \mathcal{C}$. Hence $Q$ is a $C$-quasi-closed set (w.r.t. $C$ ) and so $C+\{Q\}$ is a closure system. It contains the empty set and since every closed set of $C+\{Q\}$ is covered by a closed set containing one more element ( $C$ for $Q$ ), $C+\{Q\}$ is a convex geometry.

In the following, we denote by $\sigma$ (respectively, $\sigma^{\prime}$ ) the closure operator associated to the convex geometry $\mathcal{C}$ (respectively, $C^{\prime}$ ) and by $e x$ (respectively, $e x^{\prime}$ ) the set of extreme elements w.r.t $\sigma$ (respectively, $\sigma^{\prime}$ ). As well we set up $J_{\mathcal{C}}=J, M_{\mathcal{C}}=M$ and $J_{\mathcal{C}^{\prime}}=J^{\prime}, M_{\mathcal{C}^{\prime}}=M^{\prime}$.

Corollary 6 Let $C$ be a convex geometry on $S, Q=\bar{Q}-u\left(\right.$ with $\left.\bar{Q}=\sigma_{C}(Q)\right)$ a $\mathcal{G}$-admissible quasi-closed set for $\mathcal{C}$, and $\mathcal{C}^{\prime}=\mathcal{C}+\{Q\}$ the associated convex geometry covering $\mathcal{C}$ in $\mathcal{G}^{+}$.

For $X \subseteq S$,

$$
e x^{\prime} X= \begin{cases}\operatorname{ex} \bar{Q}+u & \text { if ex } \bar{Q}+u \subseteq X \subseteq \bar{Q} \\ \operatorname{ex} X & \text { otherwise }\end{cases}
$$

In particular, $e x^{\prime} \bar{Q}=e x \bar{Q}+u$ and $e x^{\prime} Q=e x \bar{Q}$.

For $X \subseteq S$,

$$
\sigma^{\prime}(X)= \begin{cases}Q & \text { if ex } \bar{Q} \subseteq X \subseteq Q \\ \sigma(X) & \text { otherwise. }\end{cases}
$$

In addition

$$
J^{\prime}= \begin{cases}J-\bar{Q}+Q & \text { if } \bar{Q} \in J \\ J & \text { otherwise }\end{cases}
$$

and

$$
M^{\prime}=M+Q-\left\{C \in M: C \subset Q \text { and } C^{+} \not \subset Q \text { with C maximal for these properties }\right\} .
$$

Proof:

(6), first case. By Lemma 12 it suffices to show that $e x^{\prime} \bar{Q}=e x \bar{Q}+u$, i.e. that for every $t \in S, \bar{Q}-t \in C^{\prime}$ if and only if $(t \in e x \bar{Q}$ or $t=u)$. But $\bar{Q}-t \in C^{\prime}-\{Q\}=C$ is equivalent to $t \in e x \bar{Q}$, and $\bar{Q}-u=Q \in C^{\prime}$ implies $u \in e x^{\prime} \bar{Q}$.

(6), second case. This is obvious if $X \in[e x C, C]$ with $C \in C^{\prime}-\{Q, \bar{Q}\}$. Now consider $X \in\left[e x^{\prime} Q, Q\right]$. We only have to show that $e x^{\prime} Q=e x \bar{Q}$. By Proposition 8 item 3 (with $\bar{Q}=C$ ), $t \in e x \bar{Q}$ implies $Q-t \in$ $C \subset C^{\prime}$, and so $e x \bar{Q} \subseteq e x^{\prime} Q$. Now consider $z \in e x^{\prime} Q$, i.e. $Q-z \in \mathcal{C}$ and $Q-z=\bar{Q}-\{u, z\} \prec Q$. In the convex geometry $C$ there exists $t \in e x \bar{Q}$ such that $Q-z=\bar{Q}-\{u, z\} \prec \bar{Q}-t \prec \bar{Q}$. Since $u \notin e x \bar{Q}, z=t$ and so $z \in e x \bar{Q}$. Finally $e x \bar{Q}=e x^{\prime} Q$. 
(7) Immediate consequence of Lemma 12 and of (6) of this corollary.

(8) Recall that in any convex geometry $\mathcal{C}$ on $S,|J|=|S|$. Assume that $\bar{Q} \in J$ so, in $\mathcal{C}^{\prime}, \bar{Q}=Q \vee \bar{Q}^{-}$(with $\bar{Q}^{-}$the unique lower cover of $\bar{Q}$ ). Then $\bar{Q} \notin J^{\prime}$ and, since all other elements of $J$ (distinct from $\bar{Q}$ ) remain in $J^{\prime}$, then $Q$ must be in $J^{\prime}$. At last if $\bar{Q} \notin J$ then obviously $J=J^{\prime}$.

(9) It is clear that $Q \in M^{\prime}$. Now let $C \in M$ contained in $Q$ such that $C^{+} \not \subset Q$ and maximal with these properties. In $C^{\prime}, C=Q \cap C^{+}$and so $C \notin M^{\prime}$.

Corollary 7 Let $\mathcal{C}$ be a convex geometry on $S$ (different from $2^{S}$ ). For every $C \in C$ such that $C$ is a minimal dependent closed set and every $u \in C-e x C, C-u$ is a $G$-admissible quasi-closed set.

Proof: We show that $C-u$ satisfies Condition 3 of Proposition 8 By definition $e x C \subseteq Q=C-u$. Let $t \in e x C$. Then $C-t \in \mathcal{C}$ and is free. So $Q-t=C-\{t, u\} \in \mathcal{C}$.

\section{Remark:}

1. The implication $5 \Rightarrow 1$ of Proposition 8, item 6 of Corollary 6 and Corollary 7 are Theorem 2.2. and Lemma 2.3. in Johnson and Dean [23].

2. In other terms, the transformation made when we go from the convex geometry $C$ to a convex geometry $\mathcal{C}+\{Q\}$ covering $\mathcal{C}$ is the following: the Boolean interval $\mathcal{B}=[\operatorname{ex} \bar{Q}, \bar{Q}]$ is partitioned into the two Boolean intervals $[\operatorname{ex} \bar{Q}, Q]$ and $[A, \bar{Q}]$, where $Q=\bar{Q}-u$ is a coatom of $\mathcal{B}$ and $A=\operatorname{ex} \bar{Q}+u$ is the atom complement of $Q$ in $\mathcal{B}$. Johnson and Dean call this transformation expansion of a "quotient" [23]. Note that this transformation is possible if and only if the coatom $Q$ is "locally quasi-closed", in the sense that its intersection with any closed set covered by $\bar{Q}=\sigma(Q)$ is in $\mathcal{C}$ (Proposition 8, item 3 or 4 ).

We now characterize the $\mathcal{G}$-deletable closed sets of a convex geometry $\mathcal{C}$, i.e. the elements $C$ of $C$ such that $C-\{C\} \in G$. Recall that the lattice associated with a convex geometry is lower locally distributive and that if $C$ is a meet-irreducible element of the lattice $C$ then $C^{+}$denotes the unique closed set covering $C$ in $C$.

Lemma 14 Let $C$ be a convex geometry on $S$ and $C$ an element of $C$ :

1. $\left(C \in M_{C}, t \in e x C\right.$ and $\left.C-t \notin M_{C}\right)$ imply $t \in e x C^{+}$.

2. $C \prec C^{\prime}=(C+u) \in C$ implies $\emptyset \subset$ ex $C^{\prime} \subseteq\left\{t \in\right.$ exC $\left.: C-t \notin M_{C}\right\}+\{u\} \subseteq$ exC $+u$.

3. $C \in M_{C}$ and $C \prec C^{+}=C+u$ imply ex $C^{+}=\left\{t \in \operatorname{exC}: C-t \notin M_{C}\right\}+\{u\} \subseteq$ exC $+u$.

\section{Proof:}

1. We use the dual form of a result proved in a Bordalo and Monjardet's paper ([6], Lemma 8): let $C-t \prec C$ and $C-t \prec H$ be three elements of the lower locally distributive lattice $C$. Then there exists $L$ and $M \in C$ such that $C-t \prec L \prec M$ and $C \prec M$. $C \in M_{C}$ implies $M=C^{+}$and then $L=C^{+}-t$, i.e. $t \in \operatorname{exC}^{+}$. 
2. Let $t$ be in $e x C^{\prime}$. Either $t=u$ (since $u \in e x C^{\prime}$ by definition) or $t \neq u$. In this case $C \cap\left(C^{\prime}-t\right)=C-t \in \mathcal{C}$ and so $t \in e x C$ and finally $C-t \notin M_{C}$.

3. Item 1 and $u \in e x C^{+}$imply $\left\{t \in e x C: C-t \notin M_{C}\right\}+\{u\} \subseteq e x C^{+}$. Item 2 gives the converse inclusion.

In the following propositions and corollaries, we consider two convex geometries $C$ and $C^{\prime}$ on $S$. We denote by $\sigma$ (respectively, $\sigma^{\prime}$ ) their associated closure operators and by $e x$ (respectively, $e x^{\prime}$ ) the set of extreme elements w.r.t $\sigma$ (respectively, $\sigma^{\prime}$ ). As well we set up $J_{\mathcal{C}}=J, M_{\mathcal{C}}=M$ and $J_{\mathcal{C}^{\prime}}=J^{\prime}, M_{\mathcal{C}^{\prime}}=M^{\prime}$.

Proposition 9 Let $C^{\prime}$ be a convex geometry on $S$ and $C \in C^{\prime}-\{\emptyset\}$. The first three assertions are equivalent and imply the fourth one:

1. $C$ is a $\mathcal{G}$-deletable closed set, i.e. $\mathcal{C}=C^{\prime}-\{C\} \in \mathcal{G}$,

2. $C \in M^{\prime}$ and for every $G \in C^{\prime}$ with $G \prec C, G \notin M^{\prime}$,

3. $C \in M^{\prime}$ and for $C^{+}=C+u$, $e x^{\prime} C^{+}=e x^{\prime} C+u$.

4. $C \in M^{\prime}$ and $C^{+} \notin J^{\prime}$.

Moreover if $C \in J^{\prime} \cap M^{\prime}$, the four assertions are equivalent and, in this case, $\left|e x^{\prime} C^{+}\right|=2$.

\section{Proof:}

102 Since $C^{\prime}-\{C\}$ is a closure system, $C \in M^{\prime}$. Consider $G=C-t \prec C$ and assume that $G \in M^{\prime}$. Then in the convex geometry $C^{\prime}-\{C\}$ there does not exist $G+x \in C^{\prime}-\{C\}$, a contradiction.

$2 \Longrightarrow 3$ Let $C^{+}=C+u$. By lemma $14, e x^{\prime} C^{+}=\left\{t \in e x^{\prime} C: C-t \notin M^{\prime}\right\}+u \subseteq e x^{\prime} C+u$. But since, by hypothesis, for every $t \in e x^{\prime} C, C-t \notin M^{\prime}$, we get $e x^{\prime} C^{+}=e x^{\prime} C+u$.

$3 \Longrightarrow 1$ Since $C \in M^{\prime}, C^{\prime}-\{C\}$ is a closure system. To show that it is a convex geometry we show that for every $G=C-t \in C^{\prime}$, there exists $G^{\prime}=G+u(\neq C) \in C^{\prime} . t \in e x^{\prime} C$ implies $t \in e x^{\prime} C^{+}$, then $C^{+}-t=(C+u)-t \in C^{\prime}$ and $G=C-t \prec G^{\prime}=(C+u)-t=G+u$.

3 $\Longrightarrow 4$ By 33, if $t \in e x^{\prime} C, t \in e x^{\prime} C^{+}$. So $C^{+}-t \prec C$ and $C^{+}=C \vee\left(C^{+}-t\right) \notin J^{\prime}$.

At last suppose that $C \in J^{\prime} \cap M^{\prime}$ and satisfies Property 4 (i.e. $C \in M^{\prime}$ and $C^{+} \notin J^{\prime}$ ) and show that Property 3 is satisfied. Consider $t \in e x^{\prime} C^{+}$. So $C^{+}-t=H \in C^{\prime}$ and $H \cap C=C-t=C^{-} \in C^{\prime}$, which implies $t \in e x^{\prime} C$. Therefore $e x^{\prime} C^{+} \subseteq e x^{\prime} C+u$. Now since $C \in J^{\prime}, e x^{\prime} C=\{t\}$ and so $e x^{\prime} C^{+}=e x^{\prime} C+u=$ $\{t, u\}$. 
Corollary 8 Let $C^{\prime}$ be a convex geometry, $C \in M^{\prime}$ a $\mathcal{G}$-deletable closed set of $C^{\prime}$ with $C^{+}=C+u$ and $C=C^{\prime}-C \prec C^{\prime}$.

For $X \subseteq S$,

$$
\text { exX }=\left\{\begin{array}{l}
\text { ex } C \quad \text { if } e x^{\prime} C^{+} \subseteq X \subseteq C^{+} \\
e x^{\prime} X \quad \text { otherwise }
\end{array}\right.
$$

In particular $e^{+} C^{+}=e x C=e x^{\prime} C$

For $X \subseteq S$,

$$
\begin{aligned}
\sigma(X) & = \begin{cases}C^{+} & \text {if ex } x^{\prime} C \subseteq X \subseteq C \\
\sigma^{\prime}(X) & \text { otherwise. }\end{cases} \\
J & =J^{\prime} \text { if } C \notin J^{\prime} \text { and } J=J^{\prime}-\{C\}+\left\{C^{+}\right\} \text {if } C \in J^{\prime} \cap M^{\prime} \\
M & =M^{\prime}-\{C\}+\left\{G \in C^{\prime}: G \prec C \text { and } G \text { has a unique upper cover } C^{\prime} \text { distinct from } C\right\}
\end{aligned}
$$

Moreover if $G \in M-M^{\prime}$, then $C^{\prime} \prec C^{+}$.

\section{Proof:}

(10) If $X \notin\left[e x^{\prime} C^{+}, C^{+}\right], e x X=e x X^{\prime}$ is clear. In order to prove the statement when $X \in\left[e x^{\prime} C^{+}, C^{+}\right]$, it suffices (by Lemma 12 to show that $e x C^{+}=e x^{\prime} C\left(=e x^{\prime} C^{+}-u\right.$, by Proposition 9 , item 3 . Let $t$ be in $e x^{\prime} C$. Then $t \in e x^{\prime} C^{+}$and $t \neq u$. So $C^{+}-t \in C=C^{\prime}-\{C\}$, and $t \in e x C^{+}$. Conversely, if $t \in e x C^{+}, t \neq u$ since $C^{+}-u=C \notin C$. $C^{+}-t \in C$ implies $C^{+}-t \in C^{\prime}$, i.e. $t \in e x^{\prime} C^{+}-u=e x^{\prime} C$.

(11) Immediate from Lemma 12 and $(10)$.

(12) It is clear that if $G \in J^{\prime}$ (then $G \neq C^{+}$by Proposition 8 item 44) and $G \neq C$, then $G \in J$. Since $|J|=\left|J^{\prime}\right|(=|S|$, Lemma 12$)$, we get the result for $J$.

(13) $\subseteq$ : Assume that $G \in M-M^{\prime}$. Then there exists $G^{+}$such that $G^{+}$is the unique closed set in $C=C^{\prime}-\{C\}$ with $G \prec G^{+}$. Since $G \notin M^{\prime}$, we have necessarily $G \prec C=C^{+}-u$ in $C^{\prime}$. So in $C^{\prime}, G$ is covered only by $G^{+}$and $C$. Moreover, since $C \in M^{\prime}$ and $G=C-t \notin M^{\prime}$, Lemma 14 . item 1 gives $C^{+}-t \in \mathcal{C}$. But since $G=C^{+}-\{t, u\} \prec\left(C^{+}-t\right)$, we have $G^{+}=\left(C^{+}-t\right) \prec C^{+}$.

$\supseteq$ : First it is clear that if $G \in M^{\prime}$ and $G \neq C$ (so $G \nprec C$ by Proposition 9 item 2), then $G \in M$. Now consider $G \in C$ such that $\left.\{H \in C: G \prec H\}=\left\{C, C^{\prime}\right\}\right\}$. Then $G=C-t$ and, by Lemma 14 . item 1. $C^{+}-t \in C$. Since $G=C^{+}-\{t, u\} \prec C^{+}-t$ then $C^{\prime}=C^{+}-t$. So $C^{\prime}$ is the unique closed set covering $G$ in $C$, i.e. $G \in M$.

Remark: The implication $3 \Longrightarrow 1$ of Proposition 9 and Equation $(11)$ in Corollary 8 are Theorem 2.1. in [23]. It allows Johnson and Dean to propose an algorithm that constructs all the non-isomorphic convex geometries on a set with cardinality $n$.

Now using Condition 2 of Proposition 9 , we get the following result: 
Corollary 9 Every minimal meet-irreducible closed set of a convex geometry is $\mathcal{G}$-deletable.

Remark: As already said, the convex geometries are the set representations of the lower locally distributive lattices. If $\mathcal{L} \mathcal{L D}$ denotes the class of lower locally distributive lattices, an element $x$ of $L \in \mathcal{L} \mathcal{L} \mathcal{D}$ that can be deleted from $L$ such that $L-x \in \mathcal{L} \mathcal{L} \mathcal{D}$ has been called a $\mathcal{L} \mathcal{L} \mathcal{D}$-deletable element and these elements have been characterized in [6] as follows: $x$ is $\mathcal{L} \mathcal{L D}$ if and only if it satisfies (a), (b) or (c) below:

(a) either $x \in J_{L} \cap M_{L}$,

(b) or $x \in M_{L}-J_{L}$ and $\left(x^{+} \notin J_{L}\right.$ and $y \prec x$ imply $\left.y \notin M_{L}\right)$,

(c) or $x \in J_{L}-M_{L}$ and every element covering $x$ is join-irreducible.

In a convex geometry $\mathcal{C}$, a $\mathcal{G}$-deletable closed set $C$ is obviously $\mathcal{L} \mathcal{L} \mathcal{D}$-deletable (i.e. the lattice $C-\{C\}$ is in $\mathcal{L} \mathcal{L D}$ ). This corresponds to the fact that this closed set satisfies Condition 2 of Proposition 9 and conditions for being $\mathcal{L} \mathcal{L} \mathcal{D}$-deletable (a) or (b). On the contrary, the only case where a $\mathcal{L} \mathcal{L} \mathcal{D}$-deletable closed set $C$ of a convex geometry $C$ is $\mathcal{G}$-deletable is the case where $C$ satisfies Condition (b) above with $C^{+} \notin J_{C}$. Indeed in this case it is obvious that it satisfies Condition 2 of Proposition 9 . For the other cases, we can consider the following examples: take on the set $S=\{1,2,3\}$ the three convex geometries $\mathcal{C}=\{\emptyset, 1,12,123\}, C^{\prime}=\{\emptyset, 1,2,12,123\}$ and $C^{\prime \prime}=\{\emptyset, 1,12,13,123\}$, and the $\mathcal{L} \mathcal{L} \mathcal{D}$-deletable closed set 12 (for $C$ and $C^{\prime}$ ) and 1 (for $C^{\prime \prime}$ ). Then $C-12, C^{\prime}-12$ and $C^{\prime \prime}-1$ are no longer convex geometries whereas their associated lattices are still lower locally distributive.

Finally Propositions 8 and 9 above allow us to characterize the covering relation of the lattice $\mathcal{G}^{+}$:

Theorem 5 Let $C$ and $C^{\prime}$ be two convex geometries on $S$. The following conditions are equivalent:

1. $C \prec C^{\prime}$,

2. $C^{\prime}=\mathcal{C} \cup(C-u)$ with $C \in \mathcal{C}, u \in C-$ exC and for every $t \in e x C, u \in \operatorname{ex}(C-t)$,

3. $C^{\prime}=\mathcal{C} \cup(C-u)$ with $C \in \mathcal{C}, u \in C-$ exC and for every $G \prec C, u \in \operatorname{exG}$,

4. $C=C^{\prime}-\left\{C^{\prime}\right\}$ with $C^{\prime} \in M_{C^{\prime}}$ and for every $G \prec C^{\prime}, G \notin M_{C^{\prime}}$.

The following result characterizes the arrow relations on $J_{\mathcal{G}^{+}} \times M_{\mathcal{G}^{+}}$and the dependence relation $\delta$ on $J_{\mathcal{G}^{+}}$. We recall that the atoms of $\mathcal{G}^{+}$are the $n$ linear topologies associated with the $n$ linear orders on $S$ and we write $\mathcal{C}_{x_{1} \ldots x_{i} \ldots x_{n}}$ such a linear topology. Then the non empty closed sets of $\mathcal{C}_{x_{1} \ldots x_{i} \ldots x_{n}}$ are the $n$ ideals $\left\{x_{i} \ldots x_{n}\right\}$ of the linear order $x_{1}>\ldots>x_{i}>\ldots>x_{n}$ and we denote by $\left[x_{i}\right)$ such an ideal.

Proposition 10 Let $\mathcal{C}_{x_{1} x_{2} \ldots x_{n}}$ be a join-irreducible element and $\mathcal{C}_{A, x_{i}}\left(A \subseteq S, x_{i} \notin A\right)$ a meet-irreducible element of $\mathcal{G}^{+}$. The following holds:

1. $\mathcal{C}_{x_{1} x_{2} \ldots x_{n}} \downarrow \mathcal{C}_{A, x_{i}}$ if and only if $\mathcal{C}_{x_{1} x_{2} \ldots x_{n}} \not \subseteq \mathcal{C}_{A, x_{i}}$ if and only if $A \subseteq\left[x_{i+1}\right)$.

2. $\mathcal{C}_{x_{1} x_{2} \ldots x_{n}} \uparrow \mathcal{C}_{A, x_{i}}$ if and only if $\mathcal{C}_{x_{1} x_{2} \ldots x_{n}} \uparrow \mathcal{C}_{A, x_{i}}$ if and only if $A=\left[x_{i+1}\right)$.

3. $\forall \mathcal{C}_{x_{1} x_{2} \ldots x_{n}}, \mathcal{C}_{y_{1} y_{2} \ldots y_{n}} \in J_{\mathcal{G}^{+}},\left(\mathcal{C}_{x_{1} x_{2} \ldots x_{n}} \delta \mathcal{C}_{y_{1} y_{2} \ldots y_{n}} \Longleftrightarrow y_{1} \neq x_{n}\right)$. 


\section{Proof:}

1. The first assertion is true since $\mathcal{G}^{+}$is atomistic and the second one holds since we have $X \notin \mathcal{C}_{A, x_{i}}$ if and only if $X \in\left[A, S-x_{i}\right]$.

2. The fact that $\mathcal{C}_{x_{1} x_{2} \ldots x_{n}} \uparrow \mathcal{C}_{A, x_{i}}$ is equivalent to $\mathcal{C}_{x_{1} x_{2} \ldots x_{n}} \uparrow \mathcal{C}_{A, x_{i}}$ comes from the fact that $\mathcal{C}_{x_{1} x_{2} \ldots x_{n}}$ is an atom. So $\mathcal{C}_{x_{1} x_{2} \ldots x_{n}} \uparrow \mathcal{C}_{A, x_{i}}$ implies $\mathcal{C}_{x_{1} x_{2} \ldots x_{n}} \downarrow \mathcal{C}_{A, x_{i}}$ and so $A \subseteq\left[x_{i+1}\right)$. Recall that $\mathcal{C}_{A, x_{i}}$ is covered in $\mathcal{G}^{+}$by $\mathcal{C}_{A, x_{i}}^{+}=\mathcal{C}_{A, x_{i}}+\{A\}$ (Theorem 4 , item 3 and that $\mathcal{C}_{x_{1} x_{2} \ldots x_{n}} \uparrow \mathcal{C}_{A, x_{i}}$ is equivalent to $\mathcal{C}_{x_{1} x_{2} \ldots x_{n}} \vee \mathcal{C}_{A, x_{i}}=\mathcal{C}_{A, x_{i}}^{+}=\mathcal{C}_{A, x_{i}}+\{A\}$. Assume that $A \subset\left[x_{i+1}\right)$. Since $x_{i} \notin\left[x_{i+1}\right),\left[x_{i+1}\right) \notin \mathcal{C}_{A, x_{i}}$. But $\left[x_{i+1}\right) \in \mathcal{C}_{x_{1} x_{2} \ldots x_{n}}$ implies $\left[x_{i+1}\right) \in \mathcal{C}_{x_{1} x_{2} \ldots x_{n}} \vee \mathcal{C}_{A, x_{i}}=\mathcal{C}_{A, x_{i}}+\{A\}$, a contradiction.

Conversely, if $A=\left[x_{i+1}\right), A$ is the only ideal of $x_{1}>\ldots>x_{n}$ not contained in $\mathcal{C}_{A, x_{i}}$ (since $Y \notin \mathcal{C}_{A, x_{i}}$ means $\left.\left[x_{i+1}\right) \subseteq Y \subseteq S-x_{i}\right)$. Moreover, for every $X \in \mathcal{C}_{A, x_{i}}, Z=X \cap A \subseteq A$, so $Z \in \mathcal{C}_{A, x_{i}}+\{A\}$. Then $\mathcal{C}_{x_{1} x_{2} \ldots x_{n}} \vee \mathcal{C}_{A, x_{i}}=\mathcal{C}_{A, x_{i}}^{+}$, i.e. $\mathcal{C}_{x_{1} x_{2} \ldots x_{n}} \uparrow \mathcal{C}_{A, x_{i}}$.

3. $\Longrightarrow$ : By definition, $\mathcal{C}_{x_{1} x_{2} \ldots x_{n}} \delta \mathcal{C}_{y_{1} y_{2} \ldots y_{n}}$ implies the existence of $\mathcal{C}_{A, x_{i}} \in M_{\mathcal{G}^{+}}$such that $\mathcal{C}_{x_{1} x_{2} \ldots x_{n}} \uparrow$ $\mathcal{C}_{A, x_{i}}$ and $\mathcal{C}_{y_{1} y_{2} \ldots y_{n}} \nsubseteq \mathcal{C}_{A, x_{i}}$ i.e. - by items (1) and (2) - that there exists $i$ such that $A=$ $\left[x_{i+1}\right) \subseteq\left[y_{j+1}\right)$, with $y_{j}=x_{i}$. Then $y_{1} \neq x_{n}$ (if not $y_{1}=x_{n} \in A$ and, since $y_{1}>y_{j+1}, A \nsubseteq\left[y_{j+1}\right)$ ).

$\Longleftarrow$ : Assume $y_{1} \neq x_{n}$ and set $x_{i}=y_{1}$. Then $\left[x_{i+1}\right) \subseteq\left[y_{2}\right)$ and so $\mathcal{C}_{\left[x_{i+1}\right), x_{i}}$ satisfies $\mathcal{C}_{x_{1} x_{2} \ldots x_{n}} \uparrow \mathcal{C}_{\left[x_{i+1}\right), x_{i}}$ and $\mathcal{C}_{y_{1} y_{2} \ldots y_{n}} \nsubseteq \mathcal{C}_{\left[x_{i+1}\right), x_{i}}$.

Corollary 10 The lattice $\mathcal{G}^{+}$of all convex geometries on a set $S$ is simple, i.e. it admits only the two trivial congruences. In particular $\mathcal{G}^{+}$is not subdirectly decomposable.

Proof: A result by Day [13] shows that the lattice of congruences of a lattice $L$ is isomorphic to the lattice of the ideals of its dependence relation $\delta_{d}$ of $L$ (where $I$ is an ideal of $\delta_{d}$ if $x \in I$ and $y \delta_{d} x$ imply $y \in I$ ). Since $\mathcal{G}^{+}$is atomistic, $\delta_{d}=\delta$. But it immediately results from the above characterization of $\delta$ in $\mathcal{G}^{+}$ that $\delta$ admits hamiltonian cycles (take for instance the $n$ linear topologies associated to the $n$ linear orders defined by the circular permutations of the first one). Then $\delta$ has only two trivial ideals.

\section{Remark:}

1. It has been shown in [32] that the semilattice $\mathcal{G}$ of all convex geometries is isomorphic to the semilattice of all path-independent choice functions. So every result on $\mathcal{G}$ can be translated into a result on this semilattice.

2. The set $\mathcal{T}_{0}^{+}=\mathcal{G} \cap \mathcal{M}_{\cup}$ is the set of all $T_{0}$-topologies on $S$. The poset $\left(\mathcal{T}_{0}^{+}, \subseteq\right)$ with an added least element $\min \mathcal{T}_{0}^{+}$is a lattice. Using the results of previous sections, it would be easy to derive properties of this lattice. But since this lattice or the dual lattice of partial orders have already been well studied, we send the reader back to [4]. 


\section{Conclusion}

Throughout this paper we have studied several lattices of closure systems. In particular we have characterized the covering relation $\prec$ of each one of these lattices. This allows us to determine the changes that occur in the join-irreducible elements and the meet-irreducible elements of the lattices $C$ and $C^{\prime}$ when $C \prec C^{\prime}$ and when we go from $C$ to $C^{\prime}$ (or from $C^{\prime}$ to $C$ ). These results have interesting consequences. In [7], the authors have studied the set (in fact, the lattice) of all closure systems having the same poset of join-irreducible elements (up to isomorphism). For example they have characterized the posets $P$ for which the set of all ideals of $P$ is the only closure system having $P$ as poset of join-irreducible elements. It is natural to try to consider this problem for particular closure systems. For instance, what are the posets $P$ such that the set of all ideals of $P$ is the only convex geometry having $P$ as poset of join-irreducible elements? The results contained in this paper on the covering relation in the lattice of convex geometries allow us to characterize such posets. More generally, we can provide an algorithm giving all the convex geometries having the same poset of join-irreducible elements. We shall present these results in a forthcoming paper.

\section{Acknowledgements}

The authors wish to thank the anonymous referee for his careful reading and his numerous suggestions and corrections.

\section{References}

[1] M. E. Adams, P. Dwinger and J. Schmid, Maximal sublattices of finite distributive lattices, Algebra Universalis 36, 488-504, 1996.

[2] J. C. Arditti and R. Cori, Chaînes maximales de préordres, Aequationes Math. 15 (1), 49-54, 1977.

[3] W. W. Armstrong, Dependency structures of data base relationships, Information Processing 74, 580-583, North Holland, Amsterdam, 1974.

[4] M. Barbut and B. Monjardet, Ordre et Classification, Algèbre et Combinatoire, tomes I and II, Hachette, Paris, 1970.

[5] G. Birkhoff, Lattice Theory, American Mathematical Society, Providence, 1940 (1967, 3rd ed.).

[6] G. H. Bordalo and B. Monjardet, Reducible classes of finite lattices, Order 13 (4), 379-390, 1996.

[7] G. H. Bordalo and B. Monjardet, The lattice of strict completions of a finite poset, Algebra Universalis 47, 183-200, 2002.

[8] G. Burosch, J. Demetrovics and G. Katona, The poset of closures as model of changing databases, Order 4, 30-59, 1987.

[9] N. Caspard and B. Monjardet, The lattices of closure systems, closure operators and implicational systems on a finite set: a survey, Discrete Applied Mathematics, 127 (2), 241-269, 2003. 
[10] C. C. Chen, K. M. Koh and S. K. Tan, Frattini sublattices of distributive lattices, Algebra Universalis 3 (3), 294-303, 1973.

[11] C. Chameni-Nembua and B. Monjardet, Les treillis pseudo-complémentés finis, Europ. J. Combinatorics vol.13, 89-107, 1992.

[12] B. A. Davey and H. A. Priestley, Introduction to lattices and order, Cambridge University Press, Cambridge, 1990.

[13] A. Day, Characterization of finite lattices that are bounded-homomorphic images or sublattices of free lattices, Canad. J. Math. 31, 69-78, 1979.

[14] J. Demetrovics, L. O. Libkin and I. B. Muchnik, Functional dependencies in relational databases: a lattice point of view, Discrete Applied Mathematics 40, 155-185, 1992.

[15] P. H. Edelman and R. E. Jamison, The theory of convex geometries, Geometriae Dedicata 19, 247$270,1985$.

[16] P. H. Edelman, Abstract convexity and meet-distributive lattices, Contemporary Mathematics 57, 127-150, 1986.

[17] P. H. Edelman and M. E. Saks, Combinatorial representations and convex dimension of convex geometries, Order 5 (1), 23-32, 1988.

[18] M. Erné and J. Rheinold, Intervals in Lattices of Quasiorders, Order 12, 375-403, 1995.

[19] R. Freese, J. Jezek and J. B. Nation, Free lattices, Mathematical Surveys and Monographs, vol. 42, American Mathematical Society, 1991.

[20] B. Ganter, Two basic algorithms in concept analysis, report 831 TH Darmstadt, FB Mathematik 1984; published as Algorithmen zur formalen Begriffsanalyse, in Beitraege zur Begriffsanalyse, (B. Ganter, R. Wille and K.E. Wolff, eds), Wissenchaftverlag, Mannheim (1987) 241-254.

[21] B. Ganter and R. Wille, Formal concept analysis, Mathematical foundations, Springer Verlag, Berlin (1999).

[22] L. Guigues and V. Duquenne, Familles minimales d'implications informatives résultant d'un tableau de données binaires, Mathématiques et Sciences humaines 95, 5-18, 1986.

[23] M. R. Johnson and R. A. Dean, Construction of finite lower locally distributive lattices, preprint, 2002.

[24] B. Korte, L. Lovasz and R. Schrader, Greedoids, Springer Verlag, Berlin, 1991.

[25] G. A. Koshevoy, Choice functions and abstract convex geometries, Mathematical Social Sciences 38 (1), 35-44, 1999.

[26] R. E. Larson and S. J. Andima, The lattice of topologies: a survey, Rocky Mountain Journal of Mathematics, 5 (2), 177-198, 1975. 
[27] B. Leclerc and B. Monjardet, Latticial Theory of Consensus, in Social Science, Welfare and Ethics, V. Barnett, H. Moulin, M. Salles and N. Schofiels, eds, Cambridge University Press, 145-160, 1995.

[28] B. Monjardet, Treillis d'ordres, in Ordres, work for the seminary of Aix-en-Provence on the orders, Gauthier-Villars, Paris, 29-45, 1971.

[29] B. Monjardet, The consequences of Dilworth's work on lattices with unique irreducible decompositions. In: K.P. Bogart, R. Freese and J. Kung, (Eds), The Dilworth theorems Selected papers of Robert P. Dilworth. Birkhäuser, Boston, 192-201, 1990.

[30] B. Monjardet, Arrowian characterizations of latticial federation consensus functions, Mathematical Social Sciences 20 (1), 51-71, 1990.

[31] B. Monjardet and N. Caspard, On a dependance relation in finite lattices, Discrete Mathematics 165/166, 497-505, 1997.

[32] B. Monjardet and V. Raderanirina, The duality between the anti-exchange closure operators and the path independent choice operators on a finite set, Mathematical Social Sciences 41 (2), 131-150, 2001 .

[33] B. Monjardet and V. Raderanirina, Lattices of choice functions and consensus problems, 2003. To appear.

[34] O. re, Some studies on closure relations, Duke Mathematical Journal 10, 761-785, 1983.

[35] I. Rival, Maximal sublattices of finite distributive lattices, Proc. Amer. Math. Soc. 37, 417-420, 1973.

[36] I. Rival, Maximal sublattices of finite distributive lattices II, Proc. Amer. Math. Soc. 44, 263-268, 1974.

[37] J. Schmid, Quasiorders and sublattices of distributive lattices, Order 19, 11-34, 2002.

[38] M. Stern, Semimodular lattices, Encyclopedia of Mathematics, Cambridge University Press, Cambridge, 1999.

[39] M. Wild, A theory of finite closure spaces based on implications, Advances in Mathematics 108 (1) 118-139, 1994. 